OPEN ACCESS

Edited by:

Andrea Cavani,

UOC Coordinamento

Scientifico_National Institute for Health Migration and Poverty

(NIHMP), Italy

Reviewed by:

Giulio Cavalli,

Vita-Salute San Raffaele

University, Italy

Cristina Albanesi,

Istituto di Dermatologia Immacolata

(IRCCS), Italy

*Correspondence: Xiaoan Tao

taoxiaoa@mail.sysu.edu.cn

Specialty section:

This article was submitted to

Cytokines and Soluble

Mediators in Immunity,

a section of the journal

Frontiers in Immunology

Received: 17 April 2021

Accepted: 14 June 2021

Published: 25 June 2021

Citation:

Su $Z$ and Tao X (2021)

Current Understanding of IL-37

in Human Health and Disease.

Front. Immunol. 12:696605. doi: 10.3389/fimmu.2021.696605

\section{Current Understanding of IL-37 in Human Health and Disease}

\author{
Zhangci Su ${ }^{1,2,3}$ and Xiaoan Tao ${ }^{1,2,3^{*}}$ \\ ${ }^{1}$ Hospital of Stomatology, Sun Yat-sen University, Guangzhou, China, ${ }^{2}$ Guanghua School of Stomatology, Sun Yat-sen \\ University, Guangzhou, China, ${ }^{3}$ Guangdong Provincial Key Laboratory of Stomatology, Guangzhou, China
}

IL-37 is a recently discovered cytokine in the IL-1 family exerting broad protective effects on inflammatory diseases, autoimmune diseases, and cancer. Immune and non-immune cells produce the IL-37 precursor upon pro-inflammatory stimuli. Intracellularly, caspase-1 cleaves and activates IL-37, and its mature form binds to Smad3; this complex translocates into the nucleus where it suppresses cytokine production, consequently reducing inflammation. Extracellularly, IL-37 forms a complex with IL-18R $\alpha$ and IL-1R8 (formerly TIR8 or SIGIRR) that transduces anti-inflammatory signals by the suppression of $\mathrm{NF}-\kappa \mathrm{B}$ and MAPK and the activation of Mer-PTEN-DOK pathways. During inflammation, IL-37 suppresses the expression of several pro-inflammatory cytokine in favor to the expression of the anti-inflammatory ones by the regulation of macrophage polarization, lipid metabolism, inflammasome function, TSLP synthesis and miRNAs function. Moreover, IL-37 not only regulates the innate and acquired immunity, but also improves aging-associated immunosenescence. Furthermore, IL-37 exerts an inhibitory effect on tumor angiogenesis and metastasis, and progression. Finally, IL-37 may have a potential ability to reduce excessive inflammation since it is aberrantly expressed in patients with inflammatory diseases, autoimmune diseases, and cancer, thus, it may be used as a marker for different types of diseases. Therefore, this review provides an updated view of the role of IL-37 in human health and disease, and discusses the potential of IL-37 as a therapeutic target and biomarker in inflammatory diseases, autoimmune diseases, and cancer.

Keywords: IL-1 family, inflammation, regulatory cytokine, acquired immunity, cancer, autoimmune disease

\section{INTRODUCTION}

Human IL-37 is a newly discovered member of the IL-1 family has the ability to inhibit inflammation and immune response by inhibiting the production of pro-inflammatory cytokines, and ameliorate inflammation-induced fatigue by inducing metabolic reprogramming and limiting the metabolic effects of inflammation (1). IL-37 consists of five variants a, b, c, d and e, and it functions as an intracellular and extracellular cytokine. IL-37 is expressed and released in the cytosol in its pro-inactive form that requires cleavage to be transformed in its active form, and maturation and secretion are mediated by inflammatory caspases upon inflammasome signaling complexes (2). IL-37 was first identified in silico in 2000. The anti-inflammatory properties of IL-37 were first revealed by the group of Prof. Dinarello (3). Pro-inflammatory stimuli, including the ones triggered 
by cytokines, can induce the production of human IL-37, which is a self-protective mechanism against uncontrolled inflammation and excessive tissue damage. IL-37-deficient mice cannot be created to confirm its anti-inflammatory function since the IL-37 homologous gene has not been identified in the mouse, but it was confirmed by the generation of transgenic mice expressing the human IL-37 gene (IL-37-tg). Moreover, IL-37 not only regulates innate and acquired immunity, but also improves aging-associated immunosenescence. Furthermore, IL-37 can exert an inhibitory effect on cancer development and progression. Excellent reviews on this topic are available (4).

Importantly, altered IL-37 expression in the serum was found in patients with different inflammatory diseases, autoimmune diseases, and cancer, as shown in Figure 1. The above discoveries increase the interest on the biological role of IL-37 also from a translational perspective, but despite that, certain biological properties and its precise role in human diseases are still unclear. Consequently, in-depth studies on its ability to inhibit inflammation and immune response are needed to consider IL-37 in the treatment of certain diseases. Therefore, this review summarized the broad anti-inflammatory properties of IL-37 in inflammatory diseases, autoimmune diseases, and cancer based on the research progress in recent 5 years.

\section{IL-37 Transcripts and IL-37 Isoforms}

The gene encoding IL-37 is located on the chromosome 2q12-13 that is very close to the regulatory regions of IL-1a and IL-1 $\beta$ genes (5). This specific location may be crucial for the role of
IL-37 as an inflammatory response inhibitor (5). The specific activity and relative abundance of each of the five transcripts (IL$37 \mathrm{a}-\mathrm{e}$ ) of the human IL-37 gene are still unclear (4). IL-37b includes 5 of the 6 exons of the IL-37 gene except for exon 3 and it is the most complete, abundant, and studied among all its isoforms $(6,7)$. IL-37 isoforms $a, b$, and d share exons 4,5 , and 6 , and encode functional proteins involved in the formation of the beta-fold barrel structure essential for the extracellular functional activity of the recombinant IL-37 $(4,8)$. The IL-37 isoforms $c$ and e lack one or more of these exons, thus, they may encode nonfunctional proteins (8).

\section{REGULATION OF IL-37 EXPRESSION AND RELEASE}

IL-37 is constitutively expressed in several different human tissues and cells, which may help in the maintenance of the immune homeostasis. IL-37 in immune cells is mainly expressed in circulating monocytes, tissue macrophages, dendritic cells (DCs), tonsil B cells, and plasma cells $(4,9)$. However, the baseline levels of IL-37 transcripts in resting human blood monocytes and DCs are very low due to the presence of an instability sequence in IL-37 mRNA and its short half-life (7). Similarly, the constitutive expression of IL-37 is low or absent in IL-37-tg mice (10). However, IL-37 expression is significantly increased by certain pro-inflammatory stimuli in tissue cells (3). After stimulation, immune cells such as monocytes, DCs,

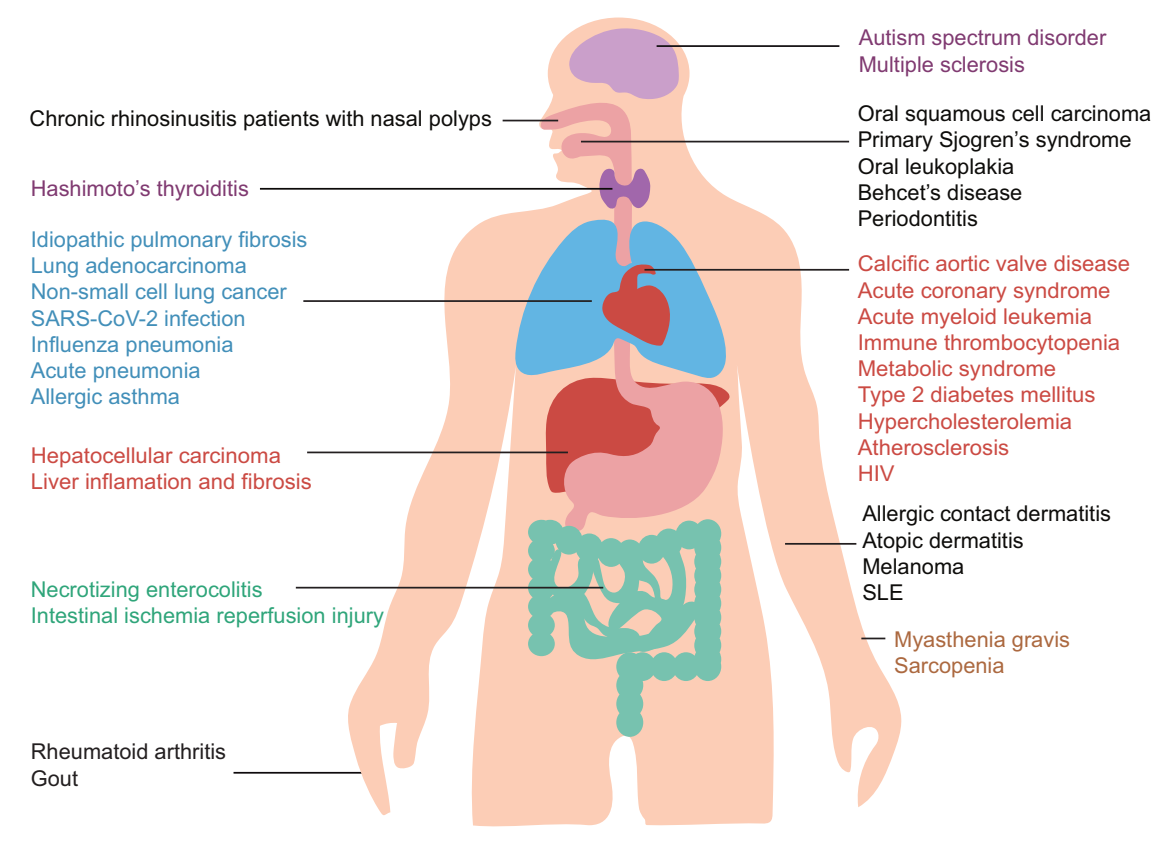

FIGURE 1 | Potential roles of IL-37 in human health and disease. IL-37 exerts a wide range of protective effects in several different diseases. Moreover, IL-37 may be used as a biomarker for inflammatory diseases, autoimmune diseases, and cancer due to the abnormal levels of IL-37 in patients affected by these diseases. 
and $\mathrm{T}$ cells express IL-37, although the vast majority of peripheral blood mononuclear cells (PBMCs) IL-37+ are monocytes (81\%-91\%) (9). To sum up, although its constitutive expression is relatively low, the inducible expression of IL-37 can exert a powerful anti-inflammatory effect or immune regulatory role.

The expression of IL-37 in autophagic cells is associated with LC3 conversion (LC3-II/I ratio) (11). In the sequence of the IL37 promoter, there are the binding motifs of AP-1 and p65 (11). Induced IL-37 expression is associated with an increase in the phosphorylated form of Erk1/2 and AP-1, and could be completely inhibited by Erk $1 / 2$ inhibitors or enhanced by Erk1/2 agonists (11). In monkeys, IL-37 expression is increased by chloroquine, and it is negatively associated with CD4 proliferation and phosphorylated STAT3 (11). Hence, upon LPS stimulation, autophagy-modifying reagents (rapamycin and chloroquine) increase the expression of IL-37 through the LC3, Erk1/2 and NF- $\mathrm{KB} / \mathrm{AP}-1$ pathways. The pro-inflammatory stimuli increase the production and secretion of IL-37 in the cytoplasm. However, IL-37 precursor does not contain the classical signal peptide at the $\mathrm{N}$-terminus that promotes its translocation into the secretory pathway (4). The presence of extracellular IL-37 precursors, the main form of extracellular IL37 , suggests that this cytokine has a release mechanism that has nothing to do with conventional secretory pathways or cell death (12). It is still unknown whether the IL-37 precursor requires the action of extracellular enzymes to form the "mature" form.

\section{IL-37-DEPENDENT MOLECULAR RESPONSES ON TARGET CELLS}

IL-37 is a dual-function cytokine since it exerts its antiinflammatory effects from its extracellular location by binding the surface membrane receptors, and from its intracellular location by the translocation into the nucleus, as shown in Figure 2.

\section{Binding of IL-37 to Receptor Complex}

The extracellular IL-37 forms a complex with IL-18 receptor $\alpha$ (IL-18R $\alpha$ ) and IL-1 receptor 8 (IL-1R8) on the cell surface, thereby transducing anti-inflammatory signals. Unlike IL-18, the binding of IL-37 to IL-18Ra does not recruit IL-18R $\beta$ chain to form a functional IL-18 receptor complex (13-16). Inversely, the orphan decoy IL-1 family receptor IL1-R8 is recruited to form the IL-37/IL-18R $\alpha /$ IL1-R8 complex which decoys MyD88 and limits signaling downstream to IL-1 family and TLR (17). IL-1R8 is necessary for IL-37 to exert its anti-inflammatory effects (1, 18-20). However, IL-1R8/Sigirr is not stable in response to IL-37 treatment (21). IL-37 induces Sigirr degradation in the ubiquitin-proteasome system through site-specific ubiquitination, which can be reversed by a deubiquitinase, USP13 (21). In a recent study, IL-37 induced activation of glycogen synthesis kinase $3 \beta$ (GSK3 $\beta$ ), which plays a role of feedback control of IL-1R8/Sigirr abundance (22). Activation of GSK3 $\beta$ promotes Sigirr phosphorylation, ubiquitination,

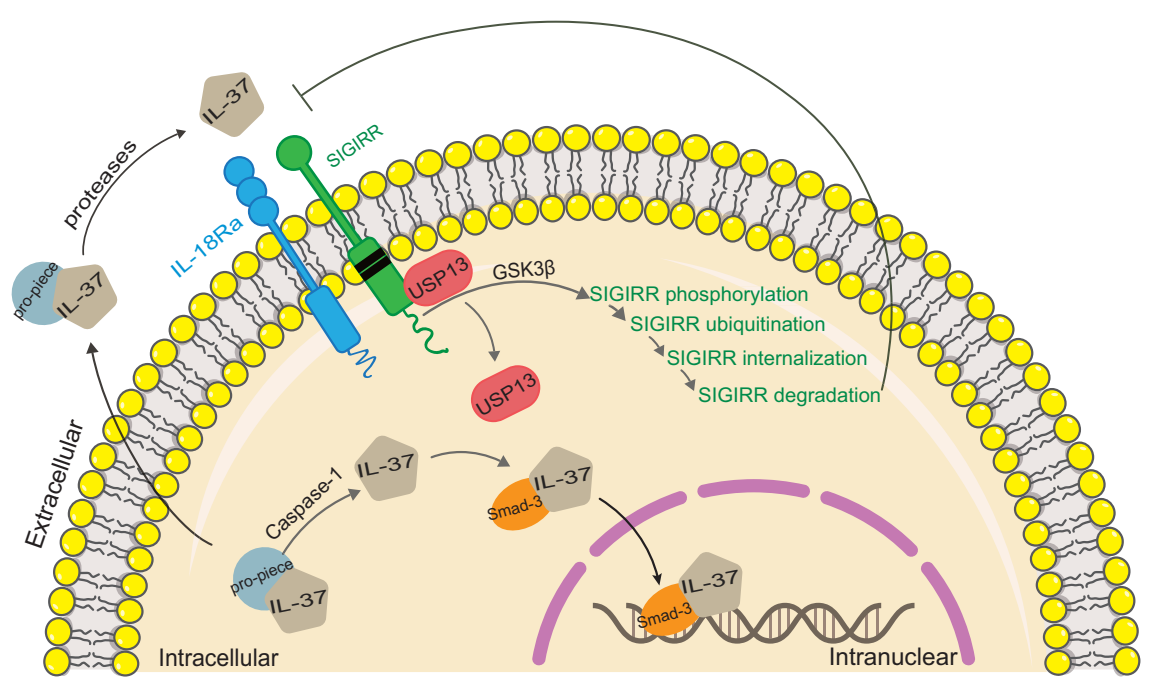

FIGURE 2 | Functional mechanisms of IL-37. IL-37 is a dual function cytokine. As regard the intracellular activity of IL-37, the pro-inflammatory stimuli increase the production of intracellular IL-37 precursor, and trigger the activation of caspase-1, which in turn cleaves IL-37 precursor into mature IL-37. Mature IL-37 binds to phosphorylated Smad-3, forming a complex that translocates into the nucleus, where it regulates gene expression. As regard the extracellular activity of IL-37, both mature and precursor forms of IL-37 are secreted by an unknown mechanism. Extracellular proteases process IL-37 precursor outside the cell, which in turn binds with IL-18R $\alpha$ and recruits IL-1R8 to form complex exerting the extracellular function of IL-37. IL-37 induced activation of GSK3 3 , which plays a role of feedback control of IL-1R8/Sigirr abundance. Activation of GSK $3 \beta$ promotes Sigirr phosphorylation, ubiquitination, internalization, and degradation through disrupting Sigirr association with USP13. 
internalization, and degradation through disrupting Sigirr association with USP13 in lung epithelial cells (22).

In addition, IL-18 binding protein (IL-18BP) can remove soluble IL-18 from the extracellular space thus preventing its binding to the receptor, consequently exerting an anti-inflammatory effect. However, the anti-inflammatory effects of IL-18BP are lost when its level increases probably because IL-18BP binds to IL-37 consequently inhibiting the anti-inflammatory effects of IL-37 itself $(15,23)$. In conclusion, IL-37 anti-inflammatory effect depends on IL-18R, IL-1R8, and IL-18BP.

Certainly, we need to be aware that IL-37 exists in two forms: a monomer of $22 \mathrm{kDa}$ and a dimer of $42 \mathrm{kDa}$ (3). The biological functions of IL-37 depends on the formation of extracellular IL37 dimers. Very low concentrations in vivo ( $1 \mu \mathrm{g}$ per mouse) of recombinant IL-37 are enough to exert its effective and ideal antiinflammatory effects $(24,25)$. However, high concentrations of IL-37 had a weak inhibitory effect on the expression of inflammatory cytokines (26). This low-dosing effectiveness of IL-37 may be related to the spontaneous formation of homodimers of IL-37. Dimers may limit the biological activity of IL-37b by reducing the steric affinity for IL-18R $\alpha$ or by blocking the recruitment of the IL-1R8 co-receptor (27), which may be considered as an auto-regulatory mechanism that limits an excessive immunosuppression. Interestingly, a single amino acid mutation in the IL-37 dimer interface leads to the formation of stable IL-37 monomers, which are maintained at high micromolar concentrations (26). Besides, the antiinflammatory activity of these IL-37 monomers in many cell types is higher than that of native IL-37 (26). Additionally, IL-37 is a heparin binding protein, and heparin secreted by mast cells blocks the biological activity of IL-37 by promoting its homodimerization $(26,28)$, suggesting that molecules selectively inhibiting the secretion of mast cell mediators can be used as new therapeutic agents together with IL-37.

\section{IL-37 Receptor-Independent Mechanisms}

Since IL-37 does not contain the nuclear localization sequence, it needs other factors to translocate into the nucleus, thereby regulating the expression of the target gene. IL-37b, c, d, and e have a caspase- 1 cleavage site at the aspartic acid (D20) of exon 1, and the caspase- 1 cleavage is necessary for IL-37 nuclear translocation $(12,29)$. After cleaved by caspase-1, the carboxyl domain of IL-37 combines with Smad3 to form a complex. The phosphorylation of Smad3 enables the translocation of IL-37 into the nucleus, where it inhibits the expression of inflammatory genes $(30,31)$.

The inhibitory effect of IL-37 on LPS-induced MAP kinase and NF- $\mathrm{KB}$ activation was reduced or lost in macrophages from IL-37-tg mice carrying the mutation of aspartic acid (D) to alanine (A) at the amino acid 20 (IL-37D20ATg) (32). However, the loss of nuclear translocation does not prevent IL-37 from exerting its anti-inflammatory effect, because IL-37D20A protein is still able to bind to its receptor, consequently exerting the inhibition of innate inflammation (32). Therefore, IL-37 can exert its anti-inflammatory effects through extracellular or intracellular mechanism, although it is still not clear which conditions and/or factors are determining the use of one mechanism instead of the other. Studies aiming at elucidating the above aspect on IL37-tg mice and humans are and will be essential to develop effective approaches in the clinical use of IL-37.

In a recent study, the protective action of IL-37 on the damage after spinal cord injury (SCI) is lost when the extracellular receptor IL-1R8 is missing, while locomotor skills and myelin sparing after SCI are significantly improved in IL-37D20ATg (33). The recombinant IL-37 protein in the presence of IL-1R8 is effective when administered in the lesion site but not systemically (33), suggesting that the nuclear translocation is not required for the beneficial effect of IL-37 on SCI damage, while the extracellular signaling of IL-37 is essential to exert a neuroprotective effect.

\section{IL-37-Dependent Intracellular Signaling}

During inflammation, IL-37 regulates the activation of various signaling phosphokinases, thereby exerting anti-inflammatory effects (3), as shown in Figure 3. IL-37 significantly reduces the activation of pro-inflammatory signaling mediators, including FAK, STAT1, mTOR, p53, p38, paxillin, Pyk2, Syk, SHP-2, and AKT (4). Moreover, anti-inflammatory mediators including the phosphatase PTEN are up-regulated in IL37-tg cells, thus inhibiting the inflammation mediated by the PK3 kinase, mTOR, MAPK and FADK pathways (34).

\section{IL-37 Genetic Variants}

Gout is a severe joint inflammation mediated by IL-1 and induced by the local accumulation of monosodium urate crystals. L37 common variants are related neither with gout nor with circulating IL- $1 \beta$ levels or IL- $1 \beta$ production by PBMCs stimulated with MSU/C16.0 (35). However, four rare IL-37 variants were found in six gout patients: p.(A144P), p. $\left(\mathrm{G} 174 \mathrm{Dfs}{ }^{\star} 16\right)$, p. $\left(\mathrm{C} 181^{\star}\right)$ and p.(N182S), but none of them was found in healthy controls (HCs) (35). These IL37 rare variants clustered in the functional domain of IL-37 in the exon 5, can lead to abnormal protein structure and function (35). The p.(N182S) variant is associated with genetic susceptibility to gout in hyperuricemic individuals of Polynesian ancestry (35). The p.(C181*) variant led to a loss of anti-inflammatory function and increased cytokine production ex vivo (35). However, the treatment with recombinant IL-37 results in the inhibition of MSU crystal-induced joint inflammation in wild type mice (35). Thus, IL-37 appears to be a relevant mediator in the pathogenesis of gout, and the recombinant form can be considered as a potential therapeutic agent to combat gouty arthritis.

\section{PATHOPHYSIOLOGICAL EFFECTS OF IL-37 ON DIFFERENT CELL TYPES}

\section{In Vitro and In Vivo Anti-Inflammatory Effects of IL-37}

Macrophage-expressed IL-37b reduces pro-inflammatory gene and protein expression upon various inflammatory stimuli 


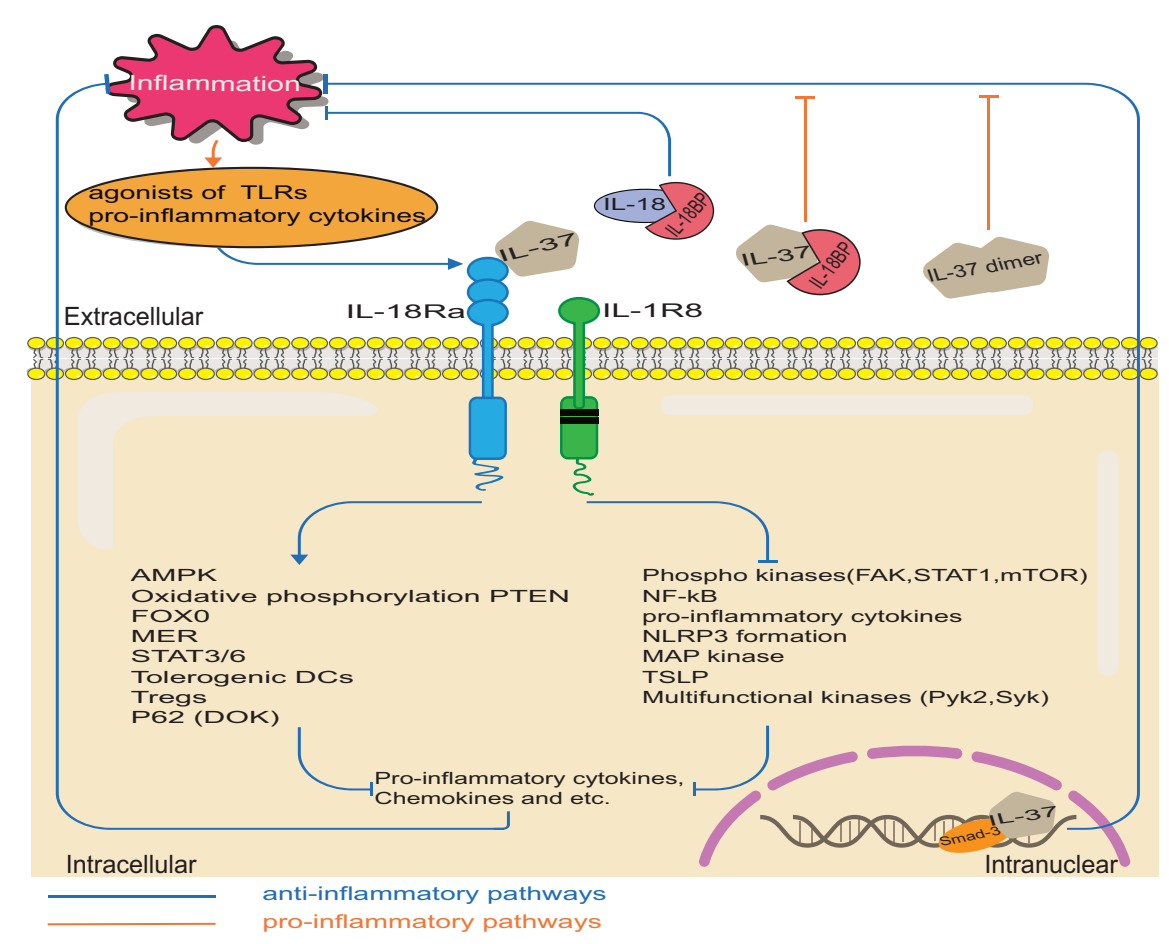

FIGURE 3 | IL-37 signaling pathways. The pro-inflammatory stimuli upregulate IL-37 expression, which in turn inhibits inflammation through various potential pathways. Intracellularly, IL-37/Smad3 complex reduces the inflammatory pathways and increases the production of anti-inflammatory cytokine. Extracellular IL-37 binds to IL-18R $\alpha / \mathrm{LL}-1 \mathrm{R} 8$. Therefore, pro-inflammatory pathways are inhibited, while anti-inflammatory pathways are activated. However, the bind of an excessive amount of IL-18BP with IL-37 reduces the anti-inflammatory activity of IL-37 and IL-18BP. Moreover, high concentrations of IL-37 had a weak inhibitory effect on the expression of inflammatory cytokines, because of the spontaneous formation of homodimers of IL-37.

relevant to atherosclerosis (36). However, knockdown of IL-37 significantly increased the LPS-induced inflammatory gene and protein expressions in WISH cells, which were reversed by administering recombinant human IL-37 (rhIL-37) (37). Similarly, administration of IL-37 neutralizing antibodies increases the production of inflammatory factors in LPSStimulated PBMCs (25). These results suggest one more time the biological function of endogenous IL-37 in the inhibition of inflammatory cytokine production.

IL-37 suppresses the production of IL- $1 \beta$ mediated by NLRP3 and AIM2 inflammasome and that of IL-18 mediated by the NLRP3 inflammasome (38). Although IL-37 does not affect the LPS-induced the expression of the mRNA of IL-18 or inflammasome components, macrophages derived from IL-37transgenic bone marrow inhibit IL-1b mRNA by up to $83 \%$ at steady-state and inhibit LPS-induced IL-1b compared with their wild-type counterparts (38). In addition, IL-37 inhibits the oligomerization/speck formation (which is a step in inflammasome activation and subsequent caspase-1 activation) and pyrolysis (-50\%) of apoptosis-associated speck-like protein containing a CARD induced by nigericin and silica (38). Moreover, IL-37d downregulates the expression of NLRP3 at the priming step through the suppression of NF- $\kappa \mathrm{B}$ activation by transcriptional profiling, and inhibits NLRP3 inflammasome activation (39). The use of Si-IL-1R8 and MCC-950, a potent and selective inhibitor of the NLRP3 inflammasome, further demonstrates the vital role of IL-1R8 and NLRP3 in the anti-inflammatory effects of IL-37 (40). Accordingly, IL-37d inhibits NLRP3 inflammasome over-activation by regulating NLRP3 transcription through a signaling pathway mediated by IL-1R8 receptor, exerting its anti-inflammatory effects through the inhibition of inflammasome activity. Thus, IL-37 can be considered as a potential agent in the treatment of inflammasome-dependent diseases.

The anti-inflammatory effect of IL-37 has been widely demonstrated not only in vitro and in IL-37 transgenic mice, but also in animal models of specific diseases $(18,20,25,41)$, when treated with IL-37. In a recent study, compared with vehicle treatment, IL-37 $(1 \mu \mathrm{g} /$ mouse) treatment of mice improves insulin sensitivity and ameliorates obesity-induced inflammation in adipose tissue after 22 weeks of high fat diet (24). Administration of IL-37 decreases plasma insulin levels and pancreatic islet mass possibly by activating AMPK and inhibiting mTOR. Thus, the anti-inflammatory effects of IL-37 can alleviate established metabolic disturbances during obesity.

Since the IL-37 precursor is processed intracellularly and extracellularly in vivo, the $\mathrm{N}$-terminus of the naturally occurring IL-37 is subjected to considerable variation, thus, its 
functional role is unclear. The administration of IL-37 isoforms with different $\mathrm{N}$-terminal ends in vivo and in vitro revealed the biological complexity of their function. For example, the administration of recombinant IL-37b with the N-terminal end at valine 46 (46-218) in vivo is more effective in suppressing inflammation compared with the original IL-37b precursor (14, 25). The same more effective effect is obtained with the use of recombinant IL-37a with the $\mathrm{N}$-terminus at lysine 27 in vitro (25). Therefore, these results highlighted the importance of identifying the amino acid sequence that provides the most effective anti-inflammatory effects to develop the most effective IL-37 therapeutic agent.

\section{Regulation of Cell Metabolism by IL-37 Balance}

Otto Heinrich Warburg was the first to describe the choice of cancer cells to obtain energy from anaerobic glycolysis instead of oxidative phosphorylation to produce more ATP necessary for a more rapid cell growth, which is indeed called the Warburg effect. Subsequently, the Warburg effect was also observed in macrophages after LPS stimulation (42), and was associated to an increased level and phosphorylation of mTOR and a decreased activity of AMPK. It was found that IL-37 can reverse the Warburg effect in target cells by inhibiting $\mathrm{mTOR}$ and activating AMPK $(3,34,43)$.

Fatigue is a common manifestation of chronic inflammatory diseases. The administration of recombinant IL-37 to mice suffering from fatigue induced by inflammation improves their resistance to exercise thanks to the activation of AMPK, which consequently induces metabolic reprogramming (1). Moreover, IL-37 treatment also markedly improved exercise tolerance in healthy mice, which was not secondary to suppression of the inflammatory response (1). These effects are related to the increased rate of oxidative phosphorylation in the mitochondria of the treated animals (1). Thus, IL-37 may be a potential target in the treatment of inflammation-induced fatigue.

Aging is related to vascular endothelial dysfunction, decreased resistance to exercise, and impaired systemic glucose metabolism. The results on experimental animals revealed that the treatment with recombinant IL-37 enhances vascular endothelial function by increasing the bioavailability of nitric oxide compared with the vehicle-treated mice (44). In addition, the treatment with recombinant IL-37 enhances the resistance to exercise by 2.4 times and the ratio of phosphorylated AMPK to AMPK (which is an indication of AMPK activation) in the quadriceps muscle by 2.9 times (44). Recombinant IL-37 treatment also improves systemic insulin sensitivity and glucose tolerance, and modifies the metabolites related to NO synthesis and fatty acid metabolism (44). Thus, IL-37 can be used as a potential agent to improve various physiological functions in the elderly people.

Cell metabolism also depends on the regulation of IL-1R8 by exogenous IL-37 (34), as suggested by the observation that the inhibition of mTOR is mediated by IL-1R8 in Th17-polarized T cells (45). In addition, the effects of IL-37 on exercise tolerance are mediated by IL-1R8 (1). Consequently, IL-37 not only exerts significant anti-inflammatory effects, but also regulates the balance of cell metabolism.

\section{Effects on Mesenchymal Stem Cells by IL-37}

Extracellular IL-37 increases the osteoblast-specific gene expression, the amount of mineral deposits, and the alkaline phosphatase activity of MSCs (46). However, the inhibitors of the $\mathrm{PI} 3 \mathrm{~K} / \mathrm{AKT}$ signaling pathway partially reduced the osteogenic differentiation of MSCs enhanced by IL-37 (46), suggesting the involvement of this pathway in the osteogenic differentiation of MSCs induced by extracellular IL-37.

IL-37 gene-modified MSCs (IL-37-MSCs) can distinctly inhibit intestinal ischemia reperfusion injury (IRI) by migrating to the damaged tissue (47). Indeed, the treatment with IL-37-MSCs on IRI rats strengthens gut barrier function and reduced the local and systemic level of the inflammatory cytokine IL-1 $\beta$, as compared with rats treated with MSCs or recombinant IL-37 (47). In addition, IL-37-MSCs treatment in IRI rats significantly decreases tissue damage due to NLRP3, the downstream targets such as cleaved caspase-1, IL-1 $\beta$, and IL-18, and IL-1 $\beta$ - and IL-18-related proinflammatory mediators IL- 6 and TNF- $\alpha$ mRNA expression, suggesting that NLRP3-related signaling pathway could be associated to the protection mediated by IL-37-MSC (47). Therefore, the modification of the IL-37 gene significantly improves the protective effect of MSCs against intestinal IRI.

\section{IL-37-Induced Effects on Macrophages}

The imbalance of M1 and M2 macrophage polarization can affect the intensity of the inflammatory responses. Calcific aortic valves present a higher amount of M1 macrophages and less IL-37 expression compared to normal valves (48). RhIL-37 downregulates the expression of inducible nitric oxide synthase, CD11c, IL-6, and monocyte chemoattractant protein 1 (MCP-1) in M1 macrophage in vitro, and inhibits their polarization through the suppression of the activation of the Notch1 and NF- $\mathrm{KB}$ pathways (48). Moreover, it up-regulates the expression of CD206 and IL-10 in M2 (48). Thus, IL-37 can shift macrophage polarization from the pro-inflammatory M1 phenotype to the anti-inflammatory M2 phenotype.

IL-37 treatment in $\mathrm{H} 1 \mathrm{~N} 1$ infected $\mathrm{BALB} / \mathrm{c}$ mice increases their survival rate and body weight, and reduces the pulmonary index, lung injury, and pro-inflammatory cytokines in the bronchoalveolar lavage fluid and lung tissue (49). Since the treatment with IL-37 increases the percentage of macrophages and IL-18R $\alpha+$ macrophages, the enhancement of the macrophage function may improve the prognosis of these mice infected with H1N1 (49). In addition, IL-37 suppresses MAPK signaling in H1N1 infected RAW264.7 cells (49). Thus, IL-37 ameliorates influenza pneumonia by the reduction of cytokine production, especially by macrophages, in a MAPK-dependent manner.

\section{IL-37-Induced Effects on Cholesterol Homeostasis}

Atherosclerosis consists of the deposition of lipids and other substances in and on the walls of arteries, thereby forming 
plaques that restrict the normal blood flow. Mature DCs exert a deleterious effect on the development of atherosclerosis. However, IL-37 suppresses the maturation of DCs induced by oxidized low-density lipoprotein, significantly increases IL-1R8 levels, and decreases TLR4 and p65 levels in vitro and in vivo (50). The treatment with IL-37 of DCs isolated from IL-1R8 and TLR4-deficient mice results in the loss of the inhibitory effect on the maturation of DCs in vitro (50). Thence, IL-37 suppresses the maturation of DCs through the IL-1R8-TLR4-NF- $\mathrm{KB}$ pathway and protects $\mathrm{ApoE}^{-/-}$mice against atherosclerosis.

IL-37 rs2708961, rs2723187, and rs2708947 polymorphisms under codominant 1 model are related to low risk of hypercholesterolemia (51). Certain polymorphisms in nonhypercholesterolemia individuals are related to the risk of having high LDL-C and glucose levels, high risk of T2DM, and low risk of having abundant visceral abdominal fat, revealing that some IL-37 polymorphisms are associated to cardiometabolic factors in both individuals with and without hypercholesterolemia (51). Furthermore, the rs2708965, rs2708962, rs6717710, rs2708961, and rs2708960 are related to high levels of C-reactive protein(CRP) in individuals with hypercholesterolemia (51). Thus, IL-37 is also able to regulate cholesterol homeostasis.

\section{Regulation of Eosinophil- and Mast Cell-Mediated Inflammatory Responses by IL-37}

IL-37 mRNA and protein expression is substantially upregulated in nasal epithelial cells of patients with chronic rhinosinusitis with nasal polyps (CRSwNP), compared with control subjects who underwent septoplasty for anatomic variations and did not have other sinonasal diseases (52). IL-37b down-regulates the expression of the TLR3 co-receptor Mex3 RNA binding family member B (Mex3B) in human nasal epithelial cells (HNECs) in vitro by the inhibition of polyinosinic-polycytidylic acid-induced production of thymic stromal lymphopoietin (TSLP); this effect is also observed in vivo in murine nasal epithelial cells (52). However, the inhibitory effect of IL-37b is abolished by the knock down or overexpression of Mex3B in BEAS-2B cells (52). The level of IL-37 is decreased in the nasal secretions of patients with eosinophilic CRSwNP, because type 2 cytokines suppress the secretion of IL-37 from HNECs (52). The level of secreted IL-37 is negatively associated to the level of Mex3B and TSLP and the eosinophil number in patients with eosinophilic CRSwNP (52). Thus, type 2 cytokines can promote Mex3B activation mediated by TLR3 and subsequent TSLP production through the inhibition of IL-37 secretion in nasal epithelial cells, thus promoting eosinophilic inflammation in patients with CRSwNP.

The interaction between human eosinophils and dermal fibroblasts triggers allergic inflammation in atopic dermatitis (AD). IL-37 levels in AD patients were significantly decreased, together with increased population of eosinophils (53). The serum concentration of involucrin, a keratinizing epithelia protein, in $\mathrm{AD}$ patients is significantly higher than that of HCs, which is related to the insufficiency of IL-37 (53). IL-37b suppresses the production of pro-inflammatory cytokines and chemokines in $\mathrm{AD}$, increases autophagosome $\mathrm{LC} 3 \mathrm{~B}$ protein biogenesis and reduces the ubiquitinated protein p62 associated with autophagy through the activation of AMPK and the inhibition of mTOR (54). In CRISPR/Cas9 human IL37b knock-in mice, IL-37b significantly alleviates the MC903induced swelling of the ear tissue and itching sensation, reduced the circulating IL- 6 and in situ inflammation, decreases eosinophil infiltration in the ear lesion, and significantly upregulates Foxp3+ regulatory $\mathrm{T}$ cells (Treg) in ear and spleen (54). Furthermore, IL-37b restores the gut microbiota diversity by the enhancement of autophagy mediated by microbiota metabolites though the regulation of the AMPK-mTOR signaling pathway (54). Thence, IL-37b can significantly reduce allergic inflammation mediated by eosinophils, as well as the diversity of intestinal bacteria and their metabolites in $\mathrm{AD}$ through the regulation of autophagy, revealing a potential therapeutic strategy against $\mathrm{AD}$.

Allergic contact dermatitis (ACD) is a $\mathrm{T}$ cell-mediated skin inflammatory disease mainly causing Erythema, vesiculation and pruritus. Mast cells (MCs) are involved in the pathogenesis of ACD. The treatment with IL-37 in ACD rats significantly reduces the swelling in their ear, the infiltration of inflammatory cell, IgE levels, the production of IL-33 and inflammatory cytokine, and inhibits MC recruitment (55). IL37 treatment in rat peritoneal mast cells from ACD rats also decreases the production of IL-6, TNF- $\alpha$, IL-13 and MCP-1 induced by IL-33 (55) and significantly inhibits NF- $\kappa B$ activation and P38 phosphorylation (55). Moreover, specific Smad3 inhibitors suppress the inhibitory effect of IL-37 on allergic inflammation mediated by MC (55), revealing the role of Smad3 in this inhibitory effect of IL-37. Thus, IL-37 with Smad3 regulation protects against inflammation induced by IL-33-regulated MC by suppressing NF- $\kappa B$ and P38 MAPK activation in ACD rats.

\section{REGULATION OF THE INNATE AND ACQUIRED IMMUNITY BY IL-37}

\section{IL-37 Regulation of Innate Immune Signaling and Trained Immunity}

Cytokines are a new immunotherapeutic method that can be potentially used in the diagnosis and treatment of various diseases. IL-37 plays a key role in innate immunity, as shown in Figure 4. For example, Intestinal epithelial cells (IEC) are in constant and direct contact with the gut microbiota, and play a central role in coordinating mucosal immunity. IEC are hyporesponsive to bacterial products, which is in part due to their strong expression of SIGIRR. In a recent study, IL-37 (100 pg/ml) attenuated FliC-induced inflammatory responses in human colonoids through inhibition of p38 and NF- $\mathrm{KB}$ signaling pathways (56). Moreover, SIGIRR mediates the inhibitory effect of IL-37 in murine colonoids (56). Therefore, IL-37 can promote IEC hypo-responsiveness by suppressing inflammatory signaling, thus regulating innate immune signaling in human and mouse colonic organoids. Zhang et al. reported that a 


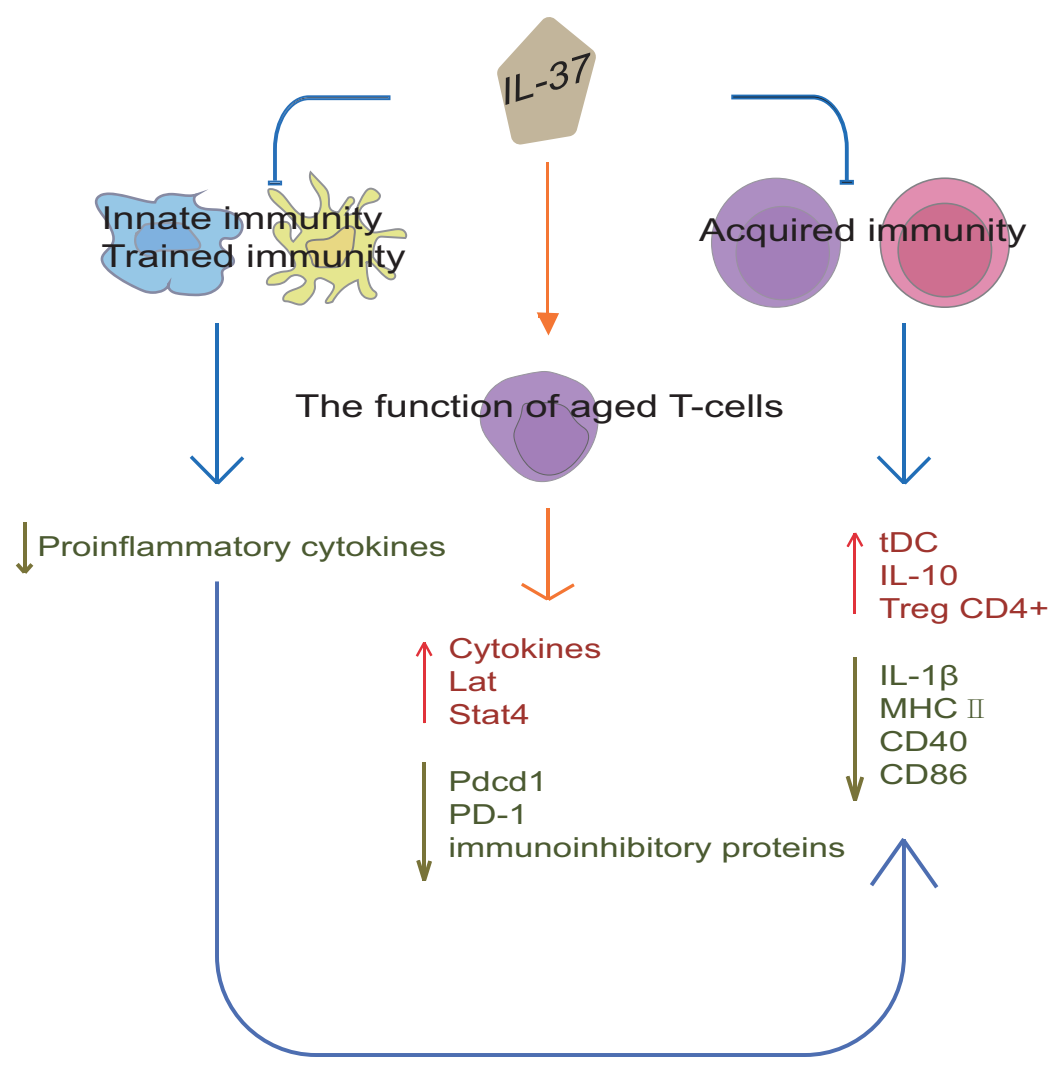

FIGURE 4 | IL-37 regulation of immunity. IL-37 can establish immunological tolerance in the gastrointestinal tract and abolish the protective effects of trained immunity by suppressing pro-inflammatory responses. Moreover, IL-37 can inhibit acquired immunity by producing tolerogenic DCs, which promote Tregs expansion and IL-10 expression, and reduce IL-1 $\beta$, CD40, CD86 and MHC II expression. The regulation of congenital inflammation by IL-37 also affects acquired immunity. Furthermore, IL-37 can promote cytokine production in aged T-cells and reduce the surface expression of programmed cell death protein 1 . IL-37 can also restore a youthful gene expression levels of Pdcd1, Lat, and Stat4 in aged T-cells, and reduce the surface expression of immunoinhibitory proteins. Thus, IL-37 not only regulates the innate and acquired immunity, but also improves aging-associated immunosenescence.

homozygous loss-of-function IL37 variant leading to infantile inflammatory bowel disease (57), which indicates that IL-37 establishes immunological tolerance in the gastrointestinal tract. In addition, IL-37 interference with the congenital protective host response against Candida albicans. The release of TNF $\alpha$ induced by Candida albicans pseudohyphae is significantly decreased in macrophages from IL-37tg mice compared with the release in macrophages from wild-type mice (58). In addition, the recruitment of neutrophils to the site of infection is suppressed in IL-37tg mice, which is associated with an increased mortality, susceptibility to disseminated candidiasis and fungal growth in the kidneys in IL-37tg mice (58). In conclusion, overexpression of IL-37 is not conducive to the early host defense against $C$. albicans in a murine model of disseminated candidiasis, so the timing of IL-37 expression in the inflamed tissue sites is vital for controlling inflammation in the host.

Trained immunity (TI) is an innate immune memory program induced in monocytes or macrophages by exposure to pathogens, microbial components, or vaccines, as a mechanism to prevent repeated infections (59-61). TI is characterized by immunometabolic changes and histone post-translational modifications, sustaining enhanced production of proinflammatory cytokines (62-64). However, excessive activation of the TI programs can cause detrimental inflammation and promote the development of inflammatory diseases $(59,65,66)$. On the one hand, administration of IL-37 in vivo reduces host pro-inflammatory responses and survival to disseminated candidiasis, thereby abolishing the protective effects of TI (67). On the other hand, IL-37 reverses the immunometabolic changes and histone post-translational modifications in monocytes, thereby inhibiting cytokine production after infection (67). Therefore, IL-37 functions as an inhibitor of TI and can be a potential therapeutic target in immune-mediated pathologies and host responses against pathogens.

\section{IL-37 Improvement of T-Cell-Mediated Immunity in Aged Backgrounds}

Aging-associated declines in immunity pose a risk for the growing aging population, while IL-37 expression levels in 
human monocytes significantly decreased with age (68). In a recent study, IL-37tg mice mitigates or prevents aging-associated chronic inflammation, splenomegaly, and accumulation of macrophages and DCs in the bone marrow and spleen (68). Moreover, IL-37 promotes cytokine production in aged T-cells and reduces the surface expression of programmed cell death protein 1 (68). IL-37 restores a youthful gene expression levels of Pdcd1, Lat, and Stat4 in aged CD4+T-cells and Lat in aged CD8+ T-cells, and reduces the surface expression of immunoinhibitory proteins (68). Recombinant IL-37 treatment not only improves T-cell function in aged mice, but also improves the efficacy of aged chimeric antigen receptor T-cells which significantly extended the survival of mice transplanted with leukemia cells (68). Thus, IL-37 can boost the function of aged T-cells and overcome aging-associated immunosenescence.

\section{IL-37 Regulation of DC Immune Function}

The number of circulating Tregs in acute coronary syndrome (ACS) patients is reduced, while the population of Th1 and Th17 is increased (69). IL-37-treated DCs obtain tolerogenic DCs (tDCs), such tDCs promote Tregs expansion and decrease the population of Th1 and Th17 when co-cultured with CD4+ T cells (69). Furthermore, IL-37-treated DCs from ACS patients are similar in phenotype and function to IL-37-treated DCs from normal coronary artery patients, and the tolerability of IL-37treated DCs is very stable (69). Hence, autologous IL-37-treated tDCs may be considered as a potential therapeutic strategy against ACS. Moreover, studies on a mouse skin sensitization model revealed that the adoptive transfer of sensitized DCs from IL37-tg mice to sensitized WT mice results in a significant decrease in immune response compared with that in mice receiving hapten-sensitized DCs from wild-type mice (70). The DCs from IL37-tg mice significantly reduce the expression of IL-1 $\beta$, IL-6, IL-12, and surface CD40 and MHC class II molecules induced by LPS in vitro, and reduce the ability to stimulate naïve $\mathrm{T}$ cells and activate antigen-specific $\mathrm{T}$ cells (70). Thus, these results suggest that IL-37 inhibits antigenspecific acquired immunity by producing tDCs, with a direct impact in the function of the acquired immune response. Furthermore, DCs isolated from the spleen of IL37-tg mice without antigen sensitization showed a sharp decrease in CD86 and MHC-II expression on the cell surface (3). Thus, the regulation of congenital inflammation by IL-37 also affects acquired immunity.

IL-10 is an important immune factor that inhibits inflammation and immune response through several mechanisms (71). A study revealed that the protective effects of IL-37 is associated to the increased expression of IL-10 in mice with acute pneumonia (70). However, other studies reported that the protective effect of IL-37 have nothing to do with IL-10 (19). Although the expression of IL-10 is increased in DCs from IL37tg mice subjected to skin sensitization, IL-37 is still able to exert its protective effects in IL-10-deficient mice (18). Thus, these inconsistent results on the potential relationship between IL-37 and IL-10 in the co-regulation of human immune responses suggest that the regulatory mechanisms still need to be clarified.

\section{Role of IL-37 in Autoimmune Diseases Systemic Lupus Erythematosus}

MSCs have immunoregulatory plasticity, and may represent a promising strategy for SLE therapy. The overexpression of IL-37 in MSCs did not change their characteristics of stem cells. These cells increase immunosuppression by inhibiting splenocyte proliferation, decreasing pro-inflammatory factors (IL-1, TNF-, IL-17, and IL-6), and inhibiting autoantibodies (anti-dsDNA and anti-ANA) (72). MSCs overexpressing IL-37 injected into the tail vein of MRL/lpr mice resulted in a better mice survival rate, less SLE signs, significantly decreased pro-inflammatory factors, less total antibody and autoantibody levels, as well as T cell number in serum and kidneys compared with mice that received only control MSCs or IL-37 treatment (72). Expression of IL-37 by MSCs can maintain high levels of serum IL-37 in the mice, and the survival time of MSCs after transplantation is prolonged probably because of the inhibitory effect of IL-37 on the inflammatory microenvironment (72). Thus, the potentiated therapeutic effect of MSCs and IL-37 is probably due to the mutually reinforcing action between them. Genetic modification to overexpress IL-37 may enhance the therapeutic effects of MSCs for SLE.

\section{Primary Sjögren's syndrome}

Patients with pSS have a higher serum IL-37 level than those in the HCs, especially pSS patients with positive anti-Ro/SSA and/ or anti-La/SSB antibodies (73). The level of total IL-18, free IL18 , and IL-18BP in the serum is higher in pSS patients than in the HCs (73). Importantly, the level of IL-37 in pSS patients is significantly and positively related to the level of antibodies including rheumatoid factor, anti-Ro/SSA and anti-La/SSB, and to the levels of total IL-18 and IL-18BP in the serum (73). Thus, IL-37 may be able to regulate the pathogenesis of pSS.

\section{Rheumatoid Arthritis}

IL-37 treatment represses the proliferation and migration and induces the apoptosis of rheumatoid arthritis fibroblast-like synoviocytes (RAFLS) (74). Indeed, the expression of apoptosisrelated proteins such as BAX and c-caspase-3 are increased, while that of $\mathrm{Bcl} 2$ and p-STAT3 are decreased in these cells (74). This increase and decrease of these proteins is regulated by STAT3 (74). Thus, IL-37 inhibits the proliferation and migration of RAFLS, and induces their apoptosis by suppressing the STAT3 pathway. Moreover, in wild-type mice subjected to Streptococcal cell wallinduced arthritis, administration of IL-37(1 $\mu \mathrm{g} / \mathrm{mouse})$ suppressed joint inflammation, which were associated with a lower recruitment of neutrophils into the joint (19). Patients with rheumatoid arthritis exhibited a markedly increased synovial expression of IL-1R8, which is required for the anti-inflammatory effects of IL-37 (19).

\section{Immune Thrombocytopenia}

ITP is an autoimmune disease characterized by low platelet count and heterogeneous bleeding, although severe bleeding in ITP is not completely correlated with low platelet count. One of the major mechanisms triggering ITP is the destruction of platelets mediated by the $\mathrm{Fc} \gamma$ receptor $(\mathrm{Fc} \gamma \mathrm{R})$. The expression 
of IL-37 is increased in the plasma of ITP patients, which is correlated with platelet count and the severity of bleeding in ITP (75). Moreover, IL-37 exerts its anti-inflammatory effects on monocytes/macrophages in ITP patients by the downregulation of the phosphorylation in the MAPK, AKT and NF- $\mathrm{KB}$ signaling pathway. IL-37 also restores the balance between activating and inhibitory Fc $\gamma$ Rs, and it reduces the antibody-mediated platelet phagocytosis by monocytes/macrophages (75). Therefore, IL-37 may be a potential biomarker to evaluate disease severity, and provide a new feasible approach in the treatment of ITP.

\section{Myasthenia Gravis}

Patients with MG possess a much lower IL-37 level in the serum and PBMCs than HCs, which is associated with severer disease (quantitative MG score), and higher follicular Th (Tfh)/Tfh17 and B cell number (76). Tfh and B cells in MG patients have high expression of the IL-37-receptor SIGIRR. IL-37 in MG patients is mainly synthesized by $\mathrm{CD} 4^{+} \mathrm{T}$ cells without overlapping with Th1, Th17, and Tfh subsets (76). In addition, regulatory IL- $37^{+} \mathrm{T}$ cells rarely express Foxp3 and CD25, while IL-4 is highly expressed (76). Furthermore, IL-37 directly bound to SIGIRR, inhibits the proliferation and cytokine production in Tfh and B cells, and the autoantibody secretion through the suppression of STAT3 signaling (76). Thus, IL-37 suppresses the autoimmunity in MG via direct target of follicular Th and B cells.

\section{Hashimoto's Thyroiditis}

Most follicular epithelial cells in tissues from HT patients express IL-37 and SIGIRR, while they are hardly expressed in infiltrating lymphocytes and other inflammatory cells (77). Moreover, IL-37 mRNA expression is significantly higher in PBMC of HT patients than in HCs (77). IL-37 pre-treatment dramatically decreases IL- $1 \beta$, TNF- $\alpha$, and MCP- 1 mRNA expression in the IFN- $\gamma$-stimulated rat thyroid cell line FRTL-5, and remarkably up-regulates IL-4 mRNA expression (77). Thus, IL-37 may have the potential to ameliorate the excessive autoimmune responses in this chronic lymphocytic thyroiditis.

\section{Multiple Sclerosis}

MS is the most common demyelinating disease of the central nervous system. Transgenic expression of IL-37 reduced inflammation and protected against neurological deficits and myelin loss in experimental autoimmune encephalomyelitis (EAE) mice by acting via IL1-R5/IL1-R8 (78). Similarly, administration of rhIL-37 exerted therapeutic actions in EAE mice (78). Although the IL-1R5/IL-1R8 receptor complex is expressed in the PBMC and brains of MS individuals, IL-37 transcripts are relatively insufficient (78). IL-37 may therefore be a potential therapeutic avenue for MS.

\section{SUPPRESSION OF CANCER BY IL-37}

\section{IL-37-Induced Effects on Tumor Angiogenesis, Migration, and Progression}

The protective effect of IL-37 against various cancer types depends on tumor type and stage and IL-37 isoforms. IL-37 treatment increases human umbilical vein endothelial cells (HUVEC) migration and tubule formation, suggesting that IL37 is a pro-angiogenic factor (79). However, the opposite effect is observed when HUVECs are treated with the supernatant from tumor cell lines overexpressing IL-37, since they undergo apoptosis and their migration and tubule formation is suppressed (79). As regard the in vivo effect, IL-37 inhibits tumor angiogenesis in the murine orthotopic hepatocellular carcinoma model, suggesting that it can induce an antiangiogenic effect (79). IL-37 reduces the expression of proangiogenic factors and increases the one of antiangiogenic factors in tumor cells (79), as well as decreases matrix metalloproteinase (MMP) 2 expression in SK-Hep-1 and SMMC-7721 cell lines overexpressing IL-37 and murine tumor models (79). The expression of MMP9 and vascular endothelial growth factor (VEGF) is also decreased in SK-Hep-1-venus and SK-Hep-1-IL-37 cells overexpressing IL-37 and murine tumors overexpressing IL-37 under hypoxic conditions (79). Moreover, tumor-associated macrophages (TAMs) promote tumor progression. PBMCs from hepatocellular carcinoma (HCC) patients show M2 polarization and a decreased IL-37 expression (80). IL-37 overexpression inhibits HCC cell proliferation, migration, and invasion by the suppression of M2 polarization through the suppression of the IL-6/STAT3 pathway (80). Furthermore, IL-37 level in HCC samples is positively associated with the infiltration degree of CD1a+ DCs (81). Indeed, IL-37 overexpression in HCC cells is related with the recruitment of more DCs into the tumor tissues by secreting high levels of specific chemokine, such as CCL3 and CCL20, significantly reducing tumor growth (81). Moreover, DCs treated with IL-37 are stimulated to secrete IL-2, IL-12, IL-12p70, IFN- $\alpha$ and IFN- $\gamma$, which indirectly enhance the anti-tumor effect of T lymphocytes (81). Thus, IL-37 may contribute to the development of additional therapeutic strategies against HCC.

IL-37b also significantly inhibits MMP and VEGF-A mRNA and protein expression in an endometriosis murine model and in in vitro uterine segments (82). In addition, a mature form of IL$37 \mathrm{~b}$ (IL-37b $\Delta 1-45$ ) effectively suppressed the migration and invasion of endometrial cancer cells by targeting the Rac1/NF$\kappa \mathrm{B} / \mathrm{MMP} 2$ signal pathway (83). Furthermore, runt related transcription factor 2 (RUNX2) is a member of the RUNX family that activates genes associated with tumorigenesis and metastasis, promoting tumor cell invasion in cancer. IL-37 overexpression significantly inhibits RUNX2 mRNA and protein expression (84), thus markedly inhibiting cell invasion.

Intracellular mature IL-37 (amino acids 46-218) effectively inhibits the migration of multiple tumor cell types through the inhibition of Rac1 activation; thus, IL-37 loss or decreased expression in lung adenocarcinoma tissues results in tumor metastasis (85). Indeed, intracellular mature IL-37 binds to the CAAX motif in the C-terminal hypervariable region of Rac1, which is normally involved in tumor angiogenesis and metastasis (85). Subsequently, this complex inhibits Rac1 membrane translocation and subsequent downstream signaling (85). Therefore, intracellular mature IL-37 may be considered as a potential therapeutic agent against Racl activity and consequent 
tumor progression. N6-methyladenosine (m6A) is a common transcriptomic modification in cancer, which is involved in the regulation of non-small cell lung cancer formation and metastasis. In a recent study, IL-37 inhibits tumor growth by regulating RNA m6A methylation in lung cancer cells, may downregulate the proliferation by inhibiting Wnt5a/5b pathway in lung cancer cells (86). Therefore, IL-37 can exert inhibitory effects on tumor angiogenesis, migration and progression, as shown in Figure 5.

\section{Protective Effect of IL-37 on Oral Squamous Cell Carcinoma}

Chronic inflammation plays a key role in the development of OSCC by the increase of inflammatory cells and cytokines. IL-37 inhibits the pro-inflammatory effects of IL-18 by binding to its receptor. Actually, OSCC patients have high levels of IL-18 and low levels of IL-37 in the serum and PBMCs (87). High IL-18 levels are related to more $\mathrm{CD} 19^{+} \mathrm{B}$ cells, while serum IL-37 is related to the decreased percentage of $\mathrm{CD} 3{ }^{+} \mathrm{CD} 8{ }^{+} \mathrm{T}$ cells (87). Therefore, serum IL-18/IL-37 balance changes the acquired immune response and affects the progression of OSCC by the modulation of the percentage of $\mathrm{CD} 19^{+} \mathrm{B}$ cells and CD3 ${ }^{+} \mathrm{CD} 8{ }^{+} \mathrm{T}$ cells. Thus, IL-37 may be considered as a potential drug against OSCC.

\section{Diagnostic Value and Prognostic Significance of IL-37 in OSCC}

The high levels of IL-18 and low levels of IL-37 in the serum and PBMC of OSCC facilitate the development of advanced tumor stage and lymph node metastasis (the odd ratios of IL-18/IL-37 is 4.903 and 12.613, respectively) (87). Non-cancer individuals can be effectively distinguished from the OSCC patients by the ratio of serum IL-18/IL-37 (cut off value: 2.15) (87). The higher the ratio of IL-18/IL-37, the shorter the overall survival and diseasefree survival in OSCC patients, despite this ratio is not an independent prognostic factor (87). Thus, the increased ratio of IL-18/IL-37 in the serum could be used as a potential biomarker for OSCC.

\section{Diagnostic Value in Oral Leukoplakia}

IL-37 expression is higher in OLK than in HCs, probably because of the inflammatory response developed in the body (88). However, IL-37 is less expressed in OLK patients without dysplasia than in those with mild/moderate dysplasia (88). IL37 overexpression in RAW264.7 cells distinctly suppresses pseudopodia, vacuolization and the expression of IL-6, TNF- $\alpha$, and IL-1 $\beta$ (88). Thus, IL-37 can be considered as a potential biomarker in the detection of oral tumorigenesis at its early stage

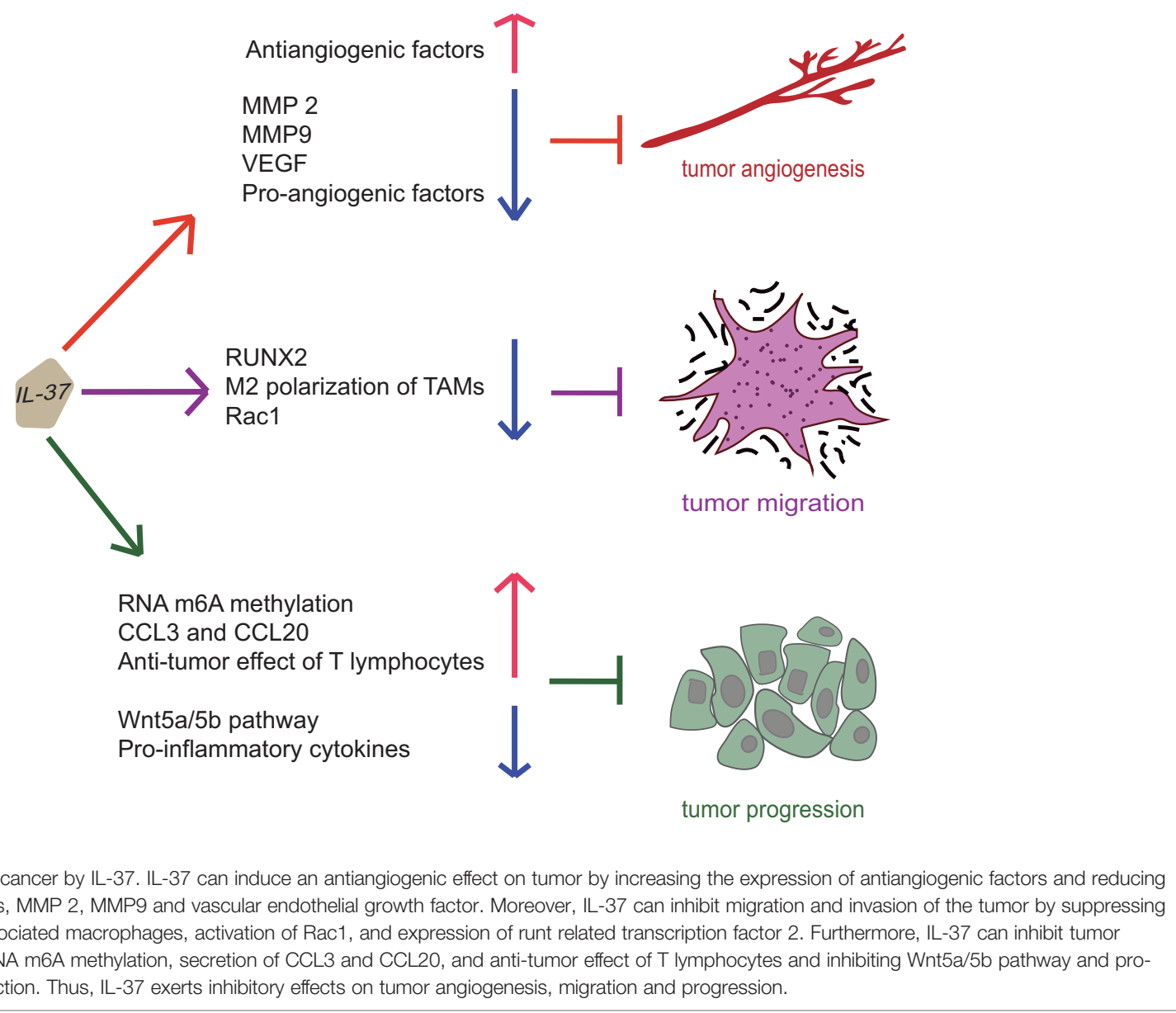


and risk assessment of the malignant transformation of premalignant lesions

\section{Melanoma}

The blood of melanoma patients contains lymphocytes (T, B, and natural killer cells) with increased IL-37 mRNA expression, with the highest expression in Treg cells (89). Similarly, Treg cells cultured in melanoma-conditioned media also express IL-37 mRNA and protein (89). In addition, the IL-1mediated secretome from human melanoma cells, particularly TGF- $\beta$, induces IL-37 mRNA expression in human Treg cells (89). Thus, the high expression of IL-37 in specific lymphocyte populations can be considered as a biomarker for immunosuppression induced by tumor.

\section{Acute Myeloid Leukemia}

AML is a malignant hematologic neoplasm, characterized by aberrant proliferation and bone marrow infiltration of myeloid progenitor cells. Serum IL-37 expression was significantly downregulated in newly diagnosed AML patients compared with HCs, and restored in patients in complete remission (90). IL-37 expression was significantly associated with nucleophosmin mutation in AML Patients, and was negatively correlated with IL-6 expression (90). Low IL-37 expression predicted poor prognosis in AML (90). Thus, IL-37 is involved in AML through regulating IL-6 expression.

\section{IL-37 INVOLVEMENT IN INFLAMMATORY SYSTEMIC DISEASES AND INFECTIONS}

\section{Necrotizing Enterocolitis}

$\mathrm{NEC}$ is a serious and currently incurable intestinal disease mainly affecting preterm infants and it is due to poorly characterized inflammatory pathways. Human and murine NEC intestines have a predominance of type $3 / \mathrm{TH} 17$ polarization and the expression of intestinal TLRs is dysregulated (91). Moreover, IL-37 and IL-1R8 are decreased in human NEC epithelia, and IL37 is also reduced in blood monocytes from infants with NEC and/or low birthweight (91). Although exogenous IL-37 is only moderately effective, IL-37tg mice are effectively protected from intestinal damage and mortality thanks to the regulation of immune homeostasis, TLR repertoires and microbial diversity (91). Thus, type 3 cytokines, TLRs and IL-37 may be used as potential targets for additional and novel therapies to combat NEC. IL-37 alternation in diseases and result of IL-37 treatment are shown in Table 1.

\section{Autism Spectrum Disorder}

ASD in children is associated with immune dysfunction and inflammation in the brain. Indeed, IL-37, IL-18 and TNF expression is increased in the amygdala and dorsolateral prefrontal cortex of children with ASD compared to their expression in the brain of non-ASD controls (92). In the same brain areas of children with ASD, IL-18R expression is also increased, while the expression of NTR3/sortilin receptor is reduced (92). The gene expression and secretion of IL-1 $\beta$ and CXCL8 after neurotensin stimulation are inhibited in cultured human microglia from normal adult brains pretreated with hrIL37 ( 1 to $100 \mathrm{ng} / \mathrm{mL}$ ) (92). Moreover, neurotensin, IL-1 $\beta$ and TNF increase the expression of IL-37 in cultured human microglia (92). Thus, IL-37 may be used as a potential therapeutic agent to cure ASD.

\section{Temporomandibular Joint Inflammation}

The synovium and the disc of osteoarthritis patients and the articular cartilage of patients with condyle fractures are characterized by a high expression of IL-37 (93). IL-37 expression is remarkably increased in synovial fluid of patients with synovitis compared with its expression in patients with osteoarthritis and disc displacement, and it is associated with the visual analogue scale score (93). Treatment of chondrocytes with IL-37-pretreated M1-conditioned medium inhibits the expression of inflammatory cytokines (40). In addition, IL-37b inhibits the expression of pro-inflammatory factors in vitro, and exerts its anti-inflammatory role through IL-1R8 by the inhibition in the activation of p38, ERK, JNK, and NF- $\kappa B$ (93). However, IL-1R8 silencing upregulates these signals and causes inflammation (93). Furthermore, IL-37 inhibits the expression of synovial M1 markers and cartilage degeneration, and promotes the expression of M2 markers in vivo (40). Thus, IL-37 protects temporomandibular joint inflammation by inhibiting inflammation and osteoclast production. Therefore, IL-37b may be a novel and promising therapeutic agent in the treatment of this type of inflammation.

\section{Behçet's Disease}

$\mathrm{BD}$ is a chronic relapse-remitting systemic inflammatory disease with unknown etiology. The release of TSLP and IL-33 is increased in $\mathrm{BD}$ patients, and they both dominated the microenvironment in cutaneous lesions with a Th2-type inflammation (94). The expression of TSLP in BD with skin lesions is closely associated to the ratio of the transcription factors GATA3/Tbet (94). However, IL-37 exerts an inhibitory effect on TSLP-skin synthesis and restore the homeostasis (94). Although serum IL-37 level in BD patients is not significantly different from its level in HCs, and is not associated with disease activity, the level of IL-37 was higher in mucocutaneous tissue than in the systemic environment (95). Thus, IL-37 may ameliorate the etiopathogenesis of $\mathrm{BD}$ by inducing more moderate clinical symptoms.

\section{Periodontitis}

Periodontitis is a common chronic inflammatory oral disease induced by the interaction between pathogen oral microorganisms and the host immune system. CD $138^{+} \mathrm{CD} 38^{+}$ plasma cells, the main immune cell type in Chronic Periodontitis gingival tissues, produce IL-35 and IL-37 (96), and experiments in vitro demonstrated that human recombinant form of these two cytokines exerts a dose-dependent inhibitory effect on osteoclast formation (96). IL-37-producing plasma cells $\left(\mathrm{CD} 138^{+} \mathrm{CD} 38^{+} \mathrm{P}_{\mathrm{IL}-37}\right)$ expressing IL-37 and IL-37/ IL-35-coproducing plasma cells $\left(\mathrm{CD} 138^{+} \mathrm{CD} 38^{+} \mathrm{P}_{\mathrm{IL}-35 / \mathrm{IL}-37}\right)$ 
TABLE 1 | IL-37 alternation in diseases and result of IL-37 treatment.

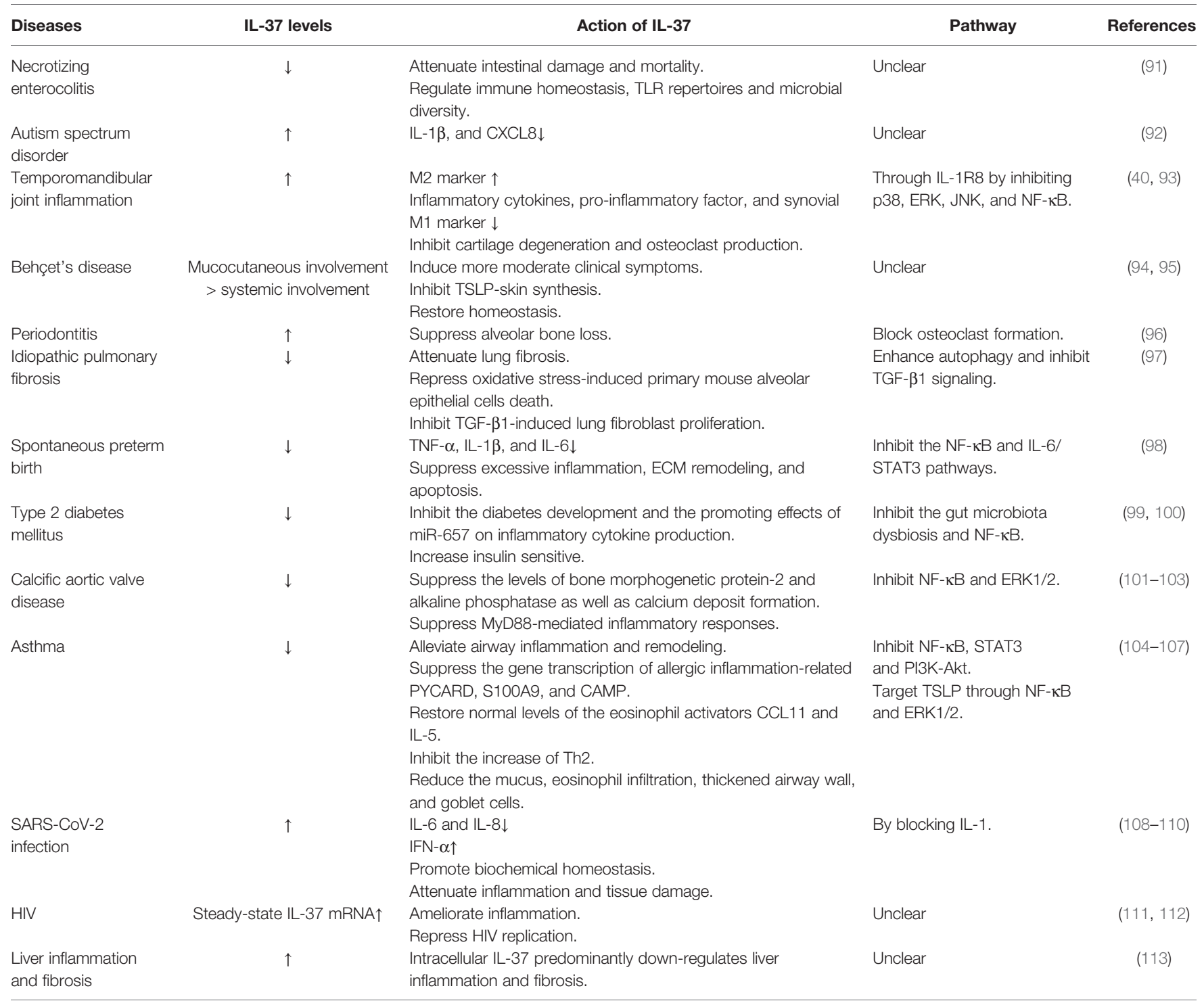

expressing both of them (96) are denoted as $\mathrm{IgG}^{+}$plasma cells, and reduce periodontitis by suppressing the loss of alveolar bone by blocking osteoclast formation (96). Thus, IL-37 and IL-35 may represent a potential therapeutic agent in the treatment of periodontitis.

\section{Idiopathic Pulmonary Fibrosis}

IL-37 protein expression is significantly reduced in alveolar epithelial cells (AECs) and alveolar macrophages in IPF patients compared with its expression in the same types of cells in HCs (97). IL-37 markedly represses mouse primary AEC death induced by oxidative stress in a dose-dependent manner, while knockdown of IL-37 markedly promotes the death of human lung cancer cells derived from AEC (A549 cells) (97). In addition, IL37 inhibits TGF- $\beta 1$ signaling and TGF- $\beta 1$-induced lung fibroblast proliferation, and enhances beclin-1-dependent autophagy in IPF fibroblasts (97). Thus, the progression of IPF may be associated to a low level of IL-37, and its ability in reducing lung fibrosis is exerted by the induction of autophagy of fibroblasts and the regulation of TGF- $\beta 1$ production.

\section{Spontaneous Preterm Birth}

The inflammatory imbalance at the maternal-fetal interface promotes the over-secretion of inflammatory factors by human amniotic epithelial cells, and induces apoptosis of human amniotic epithelial cells and extracellular matrix (ECM) degradation, leading to preterm birth. Human peripheral plasma and fetal membranes of sPTB show a decreased IL-37 expression (98). In addition, IL-37 inhibits the production of TNF- $\alpha$, IL-1 $\beta$, and IL- 6 in WISH cells (98). IL-37 silencing significantly increases LPSinduced apoptosis and activity of MMP 2 and 9 in WISH cells (98). Thus, IL-37 suppresses excessive inflammation, ECM remodeling, and apoptosis by the inhibition of TNF- $\alpha$, IL-1 $\beta$, and IL- 6 in the cells of the fetal membrane (98). 


\section{Type 2 Diabetes Mellitus}

IL-37 is highly concentrated in the serum of the insulin therapy sensitive group compared with the insulin therapy resistant group, and this high expression is associated with a less severe gut microbiota dysbiosis (99). Besides, IL-37 overexpression in model mice inhibits the dysbiosis of gut microbiota and the development of diabetes (99). Moreover, microRNAs (miRNAs) are involved in the development of gestational diabetes mellitus (GDM). The expression of miR-657 is increased, while that of IL37 is decreased in patients with GDM, thus, miR-657 is negatively associated with IL-37 (100). MiR-657 can target IL37 and regulate it and increase the proliferation of mononuclear macrophages (100). The treatment with exogenous recombinant IL-37 of LPS-induced mononuclear macrophages significantly inhibits the promoting effects of miR-657 on inflammatory cytokine production and the activation of NF- $\mathrm{KB}$ (100). On the one hand, high IL-37 levels in the serum increase insulin sensitivity in elderly type $2 \mathrm{DM}$ patients by the inhibition of the gut microbiota dysbiosis. On the other hand, IL-37 can prevent the effect of miR-657 in macrophages.

\section{Calcific Aortic Valve Disease}

Calcific aortic valve disease is a chronic inflammatory process. Aortic valve cusps (AVCu) express lower IL-37 and increased TLRs levels than mitral valve leaflets (MVL) (101), which may explain the higher incidence of calcification of AVCu than MVL. Aortic valve interstitial cells (AVICs) of diseased aortic valves express greater levels of osteogenic factors following the stimulation of TLR 2 or 4, partly due to a relative lack of IL-37 (102). Treatment of diseased AVICs with rhIL-37 suppresses the levels of bone morphogenetic protein-2 and alkaline phosphatase as well as calcium deposit formation by inhibiting NF- $\mathrm{KB}$ and ERK1/2 (102). Moreover, mice expressing human IL-37 exhibit significantly less aortic valve thickening when subjected to a TLR4 agonist or high fat diet (102). Furthermore, IL-37 suppresses MyD88-mediated inflammatory responses in AVICs (103). Thus, IL-37 has therapeutic potential for calcific aortic valve disease.

\section{Asthma}

Asthma is a common respiratory inflammatory disorder disease of childhood, characterized by airway inflammation and airway hyper-reactivity. Airway smooth muscle cells (ASMCs) play an important role in this disease. On the one hand, IL-37 alleviates airway inflammation and remodeling in ovalbumin -induced asthma via inhibiting the activation of NF- $\mathrm{KB}$ and STAT3 signalings (104). On the other hand, IL-37 not only targets TSLP through NF- $\mathrm{KB}$ and ERK1/2 signaling pathways (105), but also may act on tracheobronchial epithelial cells to inhibit fibroblasts and AMSC from producing CCL11, thereby alleviating house dust mite(HDM)-induced asthma (106).

Importantly, IL-37b significantly inhibits the production of inflammatory factors in the co-culture of human primary eosinophils and human bronchial epithelial BEAS-2B cells after the stimulation of bacterial TLR-2 ligand peptidoglycan (107). IL-37 also antagonizes the activation of NF- $\mathrm{KB}$, intracellular
PI3K-Akt, and ERK1/2, and suppresses the gene transcription of allergic inflammation-related PYCARD, S100A9, and CAMP (107). In humanized NOD/SCID mice with HDM-induced asthma, the intravenous injection of IL-37b restores normal levels of the eosinophil activators CCL11 and IL-5 in the plasma, and inhibits the increase of Th2, cytokines related to asthma such as IL-4, IL-6, and IL-13, and the level of inflammatory IL-17, CCL5, and CCL11 in lung homogenate (107). IL-37b also reduces the mucus, eosinophil infiltration, thickened airway wall, and goblet cells (107). Thus, IL-37 may exert an anti-inflammatory effect on human allergic asthma, mediated by the above signaling cascades in human eosinophils.

\section{SARS-CoV-2 Infection}

SARS-CoV-2 induces acute severe lung inflammation through IL-1, causing cytokine storm in COVID-19. SARS-CoV-2 infection is characterized by an increased level of IL-37 in the plasma (108). Higher early IL-37 response is associated with an earlier viral RNA negative status, chest CT image improvement, and cough relief compared to the patients with lower IL-37 level in the plasma (108). Moreover, higher IL-37 is associated with lower IL- 6 and IL- 8 and higher IFN- $\alpha$, and promotes biochemical homeostasis compared to the low-IL-37 group, while low IL-37 combined with high CRP and IL-8 levels predicts a poor clinical prognosis (108). IL-37 administration attenuates lung inflammation and damage of the respiratory tissue while maintaining type I IFN, consequently keeping safe the function of vital organs (108). Thus, IL-37, by blocking IL-1 $(109,110)$, may be a potential therapeutic agent to combat SARSCoV-2 infection, and IL-37, IL-8, and CRP may be used as biomarkers to recognize severe clinical cases.

\section{HIV}

HIV-1-infected patients show higher steady-state IL-37 mRNA expression in PBMCs compared to the expression in noninfected controls, and this IL-37 mRNA expression is associated with the total viral HIV-1 reservoir (111). Moreover, IL-37 in the serum is higher in treated HIV-infected patients compared with their concentration in untreated HIV-infected patients, and rhIL-37 represses HIV replication in human PHA blasts (112). Furthermore, immune cells show a decrease in the expression of IL-37 signaling co-receptor SIGIRR and its soluble form is increased in the serum in HIV-infected patients, situation that can be reversed in those patients treated with antiretroviral drugs (112). The soluble SIGIRR inhibits the anti-inflammatory effects of IL-37, and soluble SIGIRR and IL-37 concentration is associated with certain clinical parameters of the patients (112). Thus, IL-37/SIGIRR axis is functionally compromised in HIV patients and targeting it may ameliorate inflammation and reduce HIV replication in infected patients.

\section{Sarcopenia}

Sarcopenia, characterized by loss of muscle mass and functions, is a highly prevalent condition associated with inflammation in elderly individuals. Successful rehabilitation for sarcopenia results in a reduction significant of CRP $(\mathrm{p}=0.04)$ as well as of IL-18 $(\mathrm{p}=0.008)$ and IL-37 ( $\mathrm{p}=0.009)$ concentration (114). 
Thus, IL-37 may be used as biomarker to monitor the rehabilitation-associated improvement, and as therapeutic targets in sarcopenia.

\section{Liver Inflammation and Fibrosis}

IL-37tg mice improved survival, and reduced hepatic damage and liver fibrogenesis after bile duct ligation, compared with wild-type mice (113). IL-37tg mice were protected against CCl4induced liver inflammation, and colitis associated liver inflammation and fibrosis (113). Moreover, transgene IL-37 expression reduces the inflammatory response of murine hepatic stellate cells and Kupffer cells (113). IL-37 overexpression reduced the inflammatory response of IL-1 $\beta$ stimulated human LX-2 stellate cells (113). However, rhIL-37 treatment did not regulate fibrosis pathways after bile duct ligation in mice, LX2 cells or murine hepatic stellate cells (113). Therefore, intracellular IL-37 predominantly downregulates liver inflammation and fibrosis (113). Furthermore, serum IL-37 levels were positively correlated with disease severity in liver cirrhosis (113), suggesting its potential as a target regulating the course of liver fibrosis.

\section{CONCLUSIONS}

IL-37 is a dual function cytokine with both intracellular and extracellular forms that exerts broad and complex antiinflammatory and immunomodulatory effects, inhibits the excess of inflammation, and prevent tissue damage mediated by inflammation. These effects are due to the suppression in the maturation of some inflammatory cells, production of cytokines, and activation of transcription factors and signaling kinases. Extracellularly, IL-37 binds to IL-18R $\alpha$ and IL-1R8, forming a complex that transduces anti-inflammatory signals. Intracellularly, the IL-37-Smad3 complex translocates into the nucleus where it regulates the transcription, cell maturation, and cytokine production. IL-37 also regulates cell metabolism by the inhibition of mTOR and the activation of AMPK. Furthermore, IL-37 regulates innate and acquired immunity, and improves aging-associated immunosenescence. In recent years, a role for IL-37 has been discovered in several different diseases, such as

\section{REFERENCES}

1. Cavalli G, Justice JN, Boyle KE, D’Alessandro A, Eisenmesser EZ, Herrera JJ, et al. Interleukin 37 Reverses the Metabolic Cost of Inflammation, Increases Oxidative Respiration, and Improves Exercise Tolerance. Proc Natl Acad Sci USA (2017) 114(9):2313-8. doi: 10.1073/pnas.1619011114

2. Monteleone M, Stow JL, Schroder K. Mechanisms of Unconventional Secretion of IL-1 Family Cytokines. Cytokine (2015) 74(2):213-8. doi: 10.1016/j.cyto.2015.03.022

3. Nold MF, Nold-Petry CA, Zepp JA, Palmer BE, Bufler P, Dinarello CA. IL37 Is a Fundamental Inhibitor of Innate Immunity. Nat Immunol (2010) 11 (11):1014-22. doi: 10.1038/ni.1944

4. Cavalli G, Dinarello CA. Suppression of Inflammation and Acquired Immunity by IL-37. Immunol Rev (2018) 281(1):179-90. doi: 10.1111/ imr.12605 autoimmune diseases, cancer, and inflammatory diseases. The amount of IL-37 expression is different in different diseases. Thus, the potential use of IL-37 as a novel therapeutic target may be beneficial in the modulation of the inflammatory, metabolic and immune response as well as cancer development. Although IL-37tg mice are protected from the development of autoimmune diseases, IL-37 expression in these disorders is mainly high and positively associated to the disease activity. Thus, further investigations are necessary to discover the different mechanisms used by IL-37 in different autoimmune disorders. Notably, the pathophysiology of IL-37 can be understood from certain chronic infections and cancer, because they evade the immune response through host antiinflammatory and immunosuppressive mechanisms. Furthermore, IL-37 subtypes and genetic variants follow distinct underlying mechanisms, and miRNAs are also involved in the regulation of the inflammatory response via IL37 in gestational diabetes. However, the actual information on IL-37 highlights its potential role as a promising candidate in the treatment of inflammatory diseases, autoimmune diseases, and cancer. Nevertheless, clinical complications should be avoided by a strict regulation in the balance between effector immune responses required to remove pathogens and limited tissue damage caused by excessive inflammation.

\section{AUTHOR CONTRIBUTIONS}

ZS and XT contributed to the conception and design of the current study. ZS was responsible for drafting the manuscript. XT performed manuscript review. All authors contributed to the article and approved the submitted version. XT: Conceptualization, funding acquisition, investigation, methodology, resources, supervision, validation, visualization, and writing-review and editing.

\section{FUNDING}

This work was supported by grants from the National Natural Science Foundation of China (81771070).
5. Sharaf N, Nicklin MJ, di Giovine FS. Long-Range DNA Interactions at the IL-1/IL-36/IL-37 Gene Cluster (2q13) Are Induced by Activation of Monocytes. Cytokine (2014) 68(1):16-22. doi: 10.1016/j.cyto.2014.03.002

6. Sims JE, Smith DE. The IL-1 Family: Regulators of Immunity. Nat Rev Immunol (2010) 10(2):89-102. doi: 10.1038/nri2691

7. Bufler P, Gamboni-Robertson F, Azam T, Kim SH, Dinarello CA. Interleukin-1 Homologues IL-1F7b and IL-18 Contain Functional mRNA Instability Elements Within the Coding Region Responsive to Lipopolysaccharide. Biochem J (2004) 381(Pt 2):503-10. doi: 10.1042/BJ20040217

8. Kumar S, McDonnell PC, Lehr R, Tierney L, Tzimas MN, Griswold DE, et al. Identification and Initial Characterization of Four Novel Members of the Interleukin-1 Family. J Biol Chem (2000) 275(14):10308-14. doi: 10.1074/ jbc.275.14.10308

9. Rudloff I, Cho SX, Lao JC, Ngo D, McKenzie M, Nold-Petry CA, et al. Monocytes and Dendritic Cells Are the Primary Sources of Interleukin 37 in 
Human Immune Cells. J Leukoc Biol (2017) 101(4):901-11. doi: 10.1189/ jlb.3MA0616-287R

10. McNamee EN, Masterson JC, Jedlicka P, McManus M, Grenz A, Collins CB, et al. Interleukin 37 Expression Protects Mice From Colitis. Proc Natl Acad Sci USA (2011) 108(40):16711-6. doi: 10.1073/pnas.1111982108

11. Shi X, Lai C, Zhao L, Zhang M, Liu X, Peng S, et al. Chloroquine and Rapamycin Augment Interleukin-37 Expression Via the LC3, ERK, and AP1 Axis in the Presence of Lipopolysaccharides. J Immunol Res (2020) 2020:6457879. doi: 10.1155/2020/6457879

12. Bulau AM, Nold MF, Li S, Nold-Petry CA, Fink M, Mansell A, et al. Role of Caspase-1 in Nuclear Translocation of IL-37, Release of the Cytokine, and IL-37 Inhibition of Innate Immune Responses. Proc Natl Acad Sci USA (2014) 111(7):2650-5. doi: 10.1073/pnas.1324140111

13. Kumar S, Hanning CR, Brigham-Burke MR, Rieman DJ, Lehr R, Khandekar S, et al. Interleukin-1F7B (IL-1H4/IL-1F7) Is Processed by Caspase-1 and Mature IL-1F7B Binds to the IL-18 Receptor But Does Not Induce IFN-Gamma Production. Cytokine (2002) 18(2):61-71. doi: 10.1006/cyto.2002.0873

14. Pan G, Risser P, Mao W, Baldwin DT, Zhong AW, Filvaroff E, et al. IL-1H, an Interleukin 1-Related Protein That Binds IL-18 Receptor/IL-1Rrp. Cytokine (2001) 13(1):1-7. doi: 10.1006/cyto.2000.0799

15. Bufler P, Azam T, Gamboni-Robertson F, Reznikov LL, Kumar S, Dinarello CA, et al. A Complex of the IL-1 Homologue IL-1F7b and IL-18-Binding Protein Reduces IL-18 Activity. Proc Natl Acad Sci USA (2002) 99 (21):13723-8. doi: 10.1073/pnas.212519099

16. Tsutsumi N, Kimura T, Arita K, Ariyoshi M, Ohnishi H, Yamamoto T, et al. The Structural Basis for Receptor Recognition of Human Interleukin-18. Nat Commun (2014) 5:5340. doi: 10.1038/ncomms6340

17. Wald D, Qin J, Zhao Z, Qian Y, Naramura M, Tian L, et al. SIGIRR, A Negative Regulator of Toll-Like Receptor-Interleukin 1 Receptor Signaling. Nat Immunol (2003) 4(9):920-7. doi: 10.1038/ni968

18. Moretti S, Bozza S, Oikonomou V, Renga G, Casagrande A, Iannitti RG, et al. IL-37 Inhibits Inflammasome Activation and Disease Severity in Murine Aspergillosis. PloS Pathog (2014) 10(11):e1004462. doi: 10.1371/ journal.ppat.1004462

19. Cavalli G, Koenders M, Kalabokis V, Kim J, Tan AC, Garlanda C, et al. Treating Experimental Arthritis With the Innate Immune Inhibitor Interleukin-37 Reduces Joint and Systemic Inflammation. Rheumatology (Oxford) (2016) 55(12):2220-9. doi: 10.1093/rheumatology/kew325

20. Lunding L, Webering S, Vock C, Schroder A, Raedler D, Schaub B, et al. IL37 Requires IL-18Ralpha and SIGIRR/IL-1R8 to Diminish Allergic Airway Inflammation in Mice. Allergy (2015) 70(4):366-73. doi: 10.1111/all.12566

21. Li L, Wei J, Li S, Jacko AM, Weathington NM, Mallampalli RK, et al. The Deubiquitinase USP13 Stabilizes the Anti-Inflammatory Receptor IL-1R8/ Sigirr to Suppress Lung Inflammation. EBioMedicine (2019) 45:553-62. doi: 10.1016/j.ebiom.2019.06.011

22. Li L, Wei J, Suber TL, Ye Q, Miao J, Li S, et al. IL-37-Induced Activation of Glycogen Synthase Kinase $3 \beta$ Promotes IL-1R8/Sigirr Phosphorylation, Internalization, and Degradation in Lung Epithelial Cells. J Cell Physiol (2021) 236(8):5676-85. doi: 10.1002/jcp.30253

23. Banda NK, Vondracek A, Kraus D, Dinarello CA, Kim SH, Bendele A, et al. Mechanisms of Inhibition of Collagen-Induced Arthritis by Murine IL-18 Binding Protein. J Immunol (2003) 170(4):2100-5. doi: 10.4049/ jimmunol.170.4.2100

24. Ballak DB, Li S, Cavalli G, Stahl JL, Tengesdal IW, van Diepen JA, et al. Interleukin-37 Treatment of Mice With Metabolic Syndrome Improves Insulin Sensitivity and Reduces Pro-Inflammatory Cytokine Production in Adipose Tissue. J Biol Chem (2018) 293(37):14224-36. doi: 10.1074/ jbc.RA118.003698

25. Li S, Neff CP, Barber K, Hong J, Luo Y, Azam T, et al. Extracellular Forms of IL-37 Inhibit Innate Inflammation In Vitro and In Vivo But Require the IL-1 Family Decoy Receptor IL-1R8. Proc Natl Acad Sci USA (2015) 112(8):2497502. doi: $10.1073 /$ pnas. 1424626112

26. Eisenmesser EZ, Gottschlich A, Redzic JS, Paukovich N, Nix JC, Azam T, et al. Interleukin-37 Monomer Is the Active Form for Reducing Innate Immunity. Proc Natl Acad Sci USA (2019) 116(12):5514-22. doi: 10.1073/ pnas. 1819672116

27. Ellisdon AM, Nold-Petry CA, D'Andrea L, Cho SX, Lao JC, Rudloff I, et al. Homodimerization Attenuates the Anti-Inflammatory Activity of
Interleukin-37. Sci Immunol (2017) 2(8):eaaj1548. doi: 10.1126/ sciimmunol.aaj1548

28. Stevens RL, Adachi R. Protease-Proteoglycan Complexes of Mouse and Human Mast Cells and Importance of Their Beta-Tryptase-Heparin Complexes in Inflammation and Innate Immunity. Immunol Rev (2007) 217:155-67. doi: 10.1111/j.1600-065X.2007.00525.x

29. Sharma S, Kulk N, Nold MF, Graf R, Kim SH, Reinhardt D, et al. The IL-1 Family Member 7b Translocates to the Nucleus and Down-Regulates Proinflammatory Cytokines. J Immunol (2008) 180(8):5477-82. doi: 10.4049/jimmunol.180.8.5477

30. Zhao M, Li Y, Guo C, Wang L, Chu H, Zhu F, et al. IL-37 Isoform D Downregulates Pro-Inflammatory Cytokines Expression in a Smad3Dependent Manner. Cell Death Dis (2018) 9(6):582. doi: 10.1038/s41419018-0664-0

31. Grimsby S, Jaensson H, Dubrovska A, Lomnytska M, Hellman U, Souchelnytskyi S. Proteomics-Based Identification of Proteins Interacting With Smad3: SREBP-2 Forms a Complex With Smad3 and Inhibits Its Transcriptional Activity. FEBS Lett (2004) 577(1-2):93-100. doi: 10.1016/ j.febslet.2004.09.069

32. Li S, Amo-Aparicio J, Neff CP, Tengesdal IW, Azam T, Palmer BE, et al. Role for Nuclear Interleukin-37 in the Suppression of Innate Immunity. Proc Natl Acad Sci USA (2019) 116(10):4456-61. doi: 10.1073/pnas.1821111116

33. Amo-Aparicio J, Sanchez-Fernandez A, Li S, Eisenmesser EZ, Garlanda C, Dinarello CA, et al. Extracellular and Nuclear Roles of IL-37 After Spinal Cord Injury. Brain Behav Immun (2020) 91:194-201. doi: 10.1016/ j.bbi.2020.09.026

34. Nold-Petry CA, Lo CY, Rudloff I, Elgass KD, Li S, Gantier MP, et al. IL-37 Requires the Receptors IL-18R $\alpha$ and IL-1R8 (SIGIRR) to Carry Out Its Multifaceted Anti-Inflammatory Program Upon Innate Signal Transduction. Nat Immunol (2015) 16(4):354-65. doi: 10.1038/ni.3103

35. Kluck V, van Deuren RC, Cavalli G, Shaukat A, Arts P, Cleophas MC, et al. Rare Genetic Variants in Interleukin-37 Link This Anti-Inflammatory Cytokine to the Pathogenesis and Treatment of Gout. Ann Rheum Dis (2020) 79(4):536-44. doi: 10.1136/annrheumdis-2019-216233

36. Feng XX, Chi G, Wang H, Gao Y, Chen Q, Ru YX, et al. IL-37 Suppresses the Sustained Hepatic IFN- $\gamma /$ TNF- $\alpha$ Production and T Cell-Dependent Liver Injury. Int Immunopharmacol (2019) 69:184-93. doi: 10.1016/j.intimp. 2019.01.037

37. Wang L, Liu Z, Huang D, Ran Y, Zhang H, He J, et al. IL-37 Exerts AntiInflammatory Effects in Fetal Membranes of Spontaneous Preterm Birth Via the NF- $\kappa B$ and IL-6/STAT3 Signaling Pathway. Mediators Inflamm (2020) 2020:1069563. doi: $10.1155 / 2020 / 1069563$

38. Rudloff I, Ung HK, Dowling JK, Mansell A, D’Andrea L, Ellisdon AM, et al. Parsing the IL-37-Mediated Suppression of Inflammasome Function. Cells (2020) 9(1):178. doi: 10.3390/cells 9010178

39. Li Y, Chu H, Zhao M, Li C, Guan Y, Guo C, et al. IL-37d Negatively Regulates NLRP3 Transcription Via Receptor-Mediated Pathway and Alleviates DSS-Induced Colitis. Inflamm Bowel Dis (2020) 27(1):84-93. doi: $10.1093 /$ ibd/izaa124

40. Luo P, Peng S, Yan Y, Ji P, Xu J. IL-37 Inhibits M1-Like Macrophage Activation to Ameliorate Temporomandibular Joint Inflammation Through the NLRP3 Pathway. Rheumatology (Oxford) (2020) 59(10):3070-80. doi: 10.1093/rheumatology/keaa192

41. Wu B, Meng K, Ji Q, Cheng M, Yu K, Zhao X, et al. Interleukin-37 Ameliorates Myocardial Ischaemia/Reperfusion Injury in Mice. Clin Exp Immunol (2014) 176(3):438-51. doi: 10.1111/cei.12284

42. O'Neill LA, Hardie DG. Metabolism of Inflammation Limited by AMPK and Pseudo-Starvation. Nature (2013) 493(7432):346-55. doi: 10.1038/ nature11862

43. Ballak DB, van Diepen JA, Moschen AR, Jansen HJ, Hijmans A, Groenhof GJ, et al. IL-37 Protects Against Obesity-Induced Inflammation and Insulin Resistance. Nat Commun (2014) 5:4711. doi: 10.1038/ncomms5711

44. Ballak DB, Brunt VE, Sapinsley ZJ, Ziemba BP, Richey JJ, Zigler MC, et al. ShortTerm Interleukin-37 Treatment Improves Vascular Endothelial Function, Endurance Exercise Capacity, and Whole-Body Glucose Metabolism in Old Mice. Aging Cell (2020) 19(1):e13074. doi: 10.1111/acel.13074

45. Gulen MF, Kang Z, Bulek K, Youzhong W, Kim TW, Chen Y, et al. The Receptor SIGIRR Suppresses Th17 Cell Proliferation Via Inhibition of the 
Interleukin-1 Receptor Pathway and mTOR Kinase Activation. Immunity (2010) 32(1):54-66. doi: 10.1016/j.immuni.2009.12.003

46. Ye C, Zhang W, Hang K, Chen M, Hou W, Chen J, et al. Extracellular IL-37 Promotes Osteogenic Differentiation of Human Bone Marrow Mesenchymal Stem Cells Via Activation of the PI3K/AKT Signaling Pathway. Cell Death Dis (2019) 10(10):753. doi: 10.1038/s41419-019-1904-7

47. Kong D, Hu Y, Li X, Yu D, Li H, Zhao Y, et al. IL-37 Gene Modification Enhances the Protective Effects of Mesenchymal Stromal Cells on Intestinal Ischemia Reperfusion Injury. Stem Cells Int (2020) 2020:8883636. doi: 10.21203/rs.2.23662/v1

48. Zhou P, Li Q, Su S, Dong W, Zong S, Ma Q, et al. Interleukin 37 Suppresses M1 Macrophage Polarization Through Inhibition of the Notch1 and Nuclear Factor Kappa B Pathways. Front Cell Dev Biol (2020) 8:56. doi: 10.3389/ fcell.2020.00056

49. Qi F, Liu M, Li F, Lv Q, Wang G, Gong S, et al. Interleukin-37 Ameliorates Influenza Pneumonia by Attenuating Macrophage Cytokine Production in a MAPK-Dependent Manner. Front Microbiol (2019) 10:2482. doi: 10.3389/ fmicb.2019.02482

50. Liu T, Liu J, Lin Y, Que B, Chang C, Zhang J, et al. IL-37 Inhibits the Maturation of Dendritic Cells Through the IL-1R8-TLR4-NF-kappaB Pathway. Biochim Biophys Acta Mol Cell Biol Lipids (2019) 1864 (10):1338-49. doi: 10.1016/j.bbalip.2019.05.009

51. Lopez-Bautista F, Posadas-Sanchez R, Vazquez-Vazquez C, Fragoso JM, Rodriguez-Perez JM, Vargas-Alarcon G. IL-37 Gene and Cholesterol Metabolism: Association of Polymorphisms With the Presence of Hypercholesterolemia and Cardiovascular Risk Factors. The GEA Mexican Study. Biomolecules (2020) 10(10):1409. doi: 10.3390/biom10101409

52. Liu JX, Liao B, Yu QH, Wang H, Liu YB, Guo CL, et al. The IL-37-Mex3BToll-like Receptor 3 Axis in Epithelial Cells in Patients With Eosinophilic Chronic Rhinosinusitis With Nasal Polyps. J Allergy Clin Immunol (2020) 145(1):160-72. doi: 10.1016/j.jaci.2019.07.009

53. Hou T, Tsang MS, Chu IM, Kan LL, Hon KL, Leung TF, et al. Skewed Inflammation Is Associated With Aberrant Interleukin-37 Signaling Pathway in Atopic Dermatitis. Allergy (2021) 00:1-13. doi: 10.1111/all.14769

54. Hou T, Sun X, Zhu J, Hon KL, Jiang P, Chu IM, et al. IL-37 Ameliorating Allergic Inflammation in Atopic Dermatitis Through Regulating Microbiota and AMPK-mTOR Signaling Pathway-Modulated Autophagy Mechanism. Front Immunol (2020) 11:752. doi: 10.3389/fimmu.2020.00752

55. Li W, Ding F, Zhai Y, Tao W, Bi J, Fan H, et al. IL-37 Is Protective in Allergic Contact Dermatitis Through Mast Cell Inhibition. Int Immunopharmacol (2020) 83:106476. doi: 10.1016/j.intimp.2020.106476

56. Allaire JM, Poon A, Crowley SM, Han X, Sharafian Z, Moore N, et al. Interleukin-37 Regulates Innate Immune Signaling in Human and Mouse Colonic Organoids. Sci Rep (2021) 11(1):8206. doi: 10.1038/s41598-021$87592-2$

57. Zhang ZZ, Zhang Y, He T, Sweeney CL, Baris S, Karakoc-Aydiner E, et al. Homozygous IL37 Mutation Associated With Infantile Inflammatory Bowel Disease. Proc Natl Acad Sci USA (2021) 118(10):e2009217118. doi: 10.1073/ pnas. 2009217118

58. van de Veerdonk FL, Gresnigt MS, Oosting M, van der Meer JW, Joosten LA, Netea MG, et al. Protective Host Defense Against Disseminated Candidiasis Is Impaired in Mice Expressing Human Interleukin-37. Front Microbiol (2014) 5:762. doi: 10.3389/fmicb.2014.00762

59. Netea MG, Joosten LA, Latz E, Mills KH, Natoli G, Stunnenberg HG, et al. Trained Immunity: A Program of Innate Immune Memory in Health and Disease. Science (2016) 352(6284):aaf1098. doi: 10.1126/science.aaf1098

60. Netea MG, Domínguez-Andrés J, Barreiro LB, Chavakis T, Divangahi M, Fuchs E, et al. Defining Trained Immunity and Its Role in Health and Disease. Nat Rev Immunol (2020) 20(6):375-88. doi: 10.1038/s41577-0200285-6

61. Quintin J, Saeed S, Martens JHA, Giamarellos-Bourboulis EJ, Ifrim DC, Logie C, et al. Candida Albicans Infection Affords Protection Against Reinfection Via Functional Reprogramming of Monocytes. Cell Host Microbe (2012) 12(2):223-32. doi: 10.1016/j.chom.2012.06.006

62. Cheng SC, Quintin J, Cramer RA, Shepardson KM, Saeed S, Kumar V, et al. mTOR- and HIF-1 $\alpha$-Mediated Aerobic Glycolysis as Metabolic Basis for Trained Immunity. Science (2014) 345(6204):1250684. doi: 10.1126/ science. 1250684
63. Arts RJ, Novakovic B, Ter Horst R, Carvalho A, Bekkering S, Lachmandas E, et al. Glutaminolysis and Fumarate Accumulation Integrate Immunometabolic and Epigenetic Programs in Trained Immunity. Cell Metab (2016) 24(6):807-19. doi: 10.1016/j.cmet.2016.10.008

64. Arts RJ, Joosten LA, Netea MG. Immunometabolic Circuits in Trained Immunity. Semin Immunol (2016) 28(5):425-30. doi: 10.1016/ j.smim.2016.09.002

65. Mulder WJM, Ochando J, Joosten LAB, Fayad ZA, Netea MG. Therapeutic Targeting of Trained Immunity. Nat Rev Drug Discov (2019) 18(7):553-66. doi: 10.1038/s41573-019-0025-4

66. Bekkering S, Quintin J, Joosten LA, van der Meer JW, Netea MG, Riksen NP. Oxidized Low-Density Lipoprotein Induces Long-Term Proinflammatory Cytokine Production and Foam Cell Formation Via Epigenetic Reprogramming of Monocytes. Arterioscler Thromb Vasc Biol (2014) 34 (8):1731-8. doi: 10.1161/ATVBAHA.114.303887

67. Cavalli G, Tengesdal IW, Gresnigt M, Nemkov T, Arts RJW, DomínguezAndrés J, et al. The Anti-Inflammatory Cytokine Interleukin-37 Is an Inhibitor of Trained Immunity. Cell Rep (2021) 35(1):108955. doi: 10.1016/j.celrep.2021.108955

68. Hamilton JAG, Lee MY, Hunter R, Ank RS, Story JY, Talekar G, et al. Interleukin-37 Improves T-Cell-Mediated Immunity and Chimeric Antigen Receptor T-Cell Therapy in Aged Backgrounds. Aging Cell (2021) 20(2): e13309. doi: 10.1111/acel.13309

69. Mao X, Zhu R, Zhang F, Zhong Y, Yu K, Wei Y, et al. IL-37 Plays a Beneficial Role in Patients With Acute Coronary Syndrome. Mediators Inflamm (2019) 2019:9515346. doi: 10.1155/2019/9515346

70. Luo Y, Cai X, Liu S, Wang S, Nold-Petry CA, Nold MF, et al. Suppression of Antigen-Specific Adaptive Immunity by IL-37 Via Induction of Tolerogenic Dendritic Cells. Proc Natl Acad Sci USA (2014) 111(42):15178-83. doi: 10.1073/pnas.1416714111

71. Banchereau J, Pascual V, O'Garra A. From IL-2 to IL-37: The Expanding Spectrum of Anti-Inflammatory Cytokines. Nat Immunol (2012) 13 (10):925-31. doi: 10.1038/ni.2406

72. Xu J, Chen J, Li W, Lian W, Huang J, Lai B, et al. Additive Therapeutic Effects of Mesenchymal Stem Cells and IL-37 for Systemic Lupus Erythematosus. J Am Soc Nephrol (2020) 31(1):54-65. doi: 10.1681/ ASN.2019050545

73. Liuqing W, Liping X, Hui S, Jing L. Elevated IL-37, IL-18 and IL-18BP Serum Concentrations in Patients With Primary Sjogren's Syndrome. J Investig Med (2017) 65(3):717-21. doi: 10.1136/jim-2016-000301

74. Liu Y, Gao W. Interleukin-37 Inhibits Proliferation, Migration and Induces Apoptosis of Rheumatoid Arthritis Fibroblast-Like Synoviocytes (RAFLS) by Inhibiting STAT3. Xi Bao Yu Fen Zi Mian Yi Xue Za Zhi (2020) 36 (3):236-41.

75. Zhao Y, Ni X, Xu P, Liu Q, Sun T, Liu X, et al. Interleukin-37 Reduces Inflammation and Impairs Phagocytosis of Platelets in Immune Thrombocytopenia (ITP). Cytokine (2020) 125:154853. doi: 10.1016/ j.cyto.2019.154853

76. Liu Z, Zhu L, Lu Z, Chen H, Fan L, Xue Q, et al. IL-37 Represses the Autoimmunity in Myasthenia Gravis Via Directly Targeting Follicular Th and B Cells. J Immunol (2020) 204(7):1736-45. doi: 10.4049/jimmunol.1901176

77. Ren CP, Sun L, Liu FC, Zuo CL, Liu M, Gao W, et al. Potential Role of IL-37 Signaling Pathway in Feedback Regulation of Autoimmune Hashimoto Thyroiditis. Histochem Cell Biol (2019) 152(6):467-73. doi: 10.1007/ s00418-019-01820-5

78. Sánchez-Fernández A, Zandee S, Amo-Aparicio J, Charabati M, Prat A, Garlanda C, et al. IL-37 Exerts Therapeutic Effects in Experimental Autoimmune Encephalomyelitis Through the Receptor Complex IL-1R5/ IL-1R8. Theranostics (2021) 11(1):1-13. doi: 10.7150/thno.47435

79. Mei Y, Zhu Y, Teo HY, Liu Y, Song Y, Lim HY, et al. The Indirect Antiangiogenic Effect of IL-37 in the Tumor Microenvironment. J Leukoc Biol (2020) 107(5):783-96. doi: 10.1002/JLB.3MA0220-207RR

80. Zhang Z, Zhang J, He P, Han J, Sun C. Interleukin-37 Suppresses Hepatocellular Carcinoma Growth Through Inhibiting M2 Polarization of Tumor-Associated Macrophages. Mol Immunol (2020) 122:13-20. doi: 10.1016/j.molimm.2020.03.012

81. Liu Y, Zhao JJ, Zhou ZQ, Pan QZ, Zhu Q, Tang Y, et al. IL-37 Induces AntiTumor Immunity by Indirectly Promoting Dendritic Cell Recruitment and 
Activation in Hepatocellular Carcinoma. Cancer Manag Res (2019) 11:6691702. doi: 10.2147/CMAR.S200627

82. He Y, Xiong T, Guo F, Du Z, Fan Y, Sun H, et al. Interleukin-37b Inhibits the Growth of Murine Endometriosis-Like Lesions by Regulating Proliferation, Invasion, Angiogenesis and Inflammation. Mol Hum Reprod (2020) 26 (4):240-55. doi: 10.1093/molehr/gaaa014

83. Wang X, Wei Z, Tang Z, Xue C, Yu H, Zhang D, et al. IL-37b $\Delta 1-45$ Suppresses the Migration and Invasion of Endometrial Cancer Cells by Targeting the Rac1/NF-kB/MMP2 Signal Pathway. Lab Invest (2021) 101 (6):760-74. doi: 10.1038/s41374-021-00544-2

84. Ouyang P, Wu K, Su L, An W, Bie Y, Zhang H, et al. Inhibition of Human Cervical Cancer Cell Invasion by IL-37 Involving Runt Related Transcription Factor 2 Suppression. Ann Transl Med (2019) 7(20):568. doi: $10.21037 / \mathrm{atm} .2019 .09 .38$

85. Li Y, Zhao M, Guo C, Chu H, Li W, Chen X, et al. Intracellular Mature IL-37 Suppresses Tumor Metastasis Via Inhibiting Rac1 Activation. Oncogene (2018) 37(8):1095-106. doi: 10.1038/onc.2017.405

86. Mu X, Zhao Q, Chen W, Zhao Y, Yan Q, Peng R, et al. IL-37 Confers AntiTumor Activity by Regulation of m6A Methylation. Front Oncol (2020) 10:526866. doi: $10.3389 /$ fonc.2020.526866

87. Ding L, Zhao X, Zhu N, Zhao M, Hu Q, Ni Y. The Balance of Serum IL-18/ IL-37 Levels Is Disrupted During the Development of Oral Squamous Cell Carcinoma. Surg Oncol (2020) 32:99-107. doi: 10.1016/j.suronc.2019.12.001

88. Lin L, Wang J, Liu D, Liu S, Xu H, Ji N, et al. Interleukin-37 Expression and Its Potential Role in Oral Leukoplakia and Oral Squamous Cell Carcinoma. Sci Rep (2016) 6:26757. doi: 10.1038/srep26757

89. Osborne DG, Domenico J, Luo Y, Reid AL, Amato C, Zhai Z, et al. Interleukin-37 Is Highly Expressed in Regulatory T Cells of Melanoma Patients and Enhanced by Melanoma Cell Secretome. Mol Carcinog (2019) 58(9):1670-9. doi: 10.1002/mc.23044

90. Wei X, Li Y, Zhang G, Wang N, Mi M, Xin Y, et al. IL-37 Was Involved in Progress of Acute Myeloid Leukemia Through Regulating IL-6 Expression. Cancer Manag Res (2021) 13:3393-402. doi: 10.2147/CMAR.S303017

91. Cho SX, Rudloff I, Lao JC, Pang MA, Goldberg R, Bui CB, et al. Characterization of the Pathoimmunology of Necrotizing Enterocolitis Reveals Novel Therapeutic Opportunities. Nat Commun (2020) 11 (1):5794. doi: 10.1038/s41467-020-19400-w

92. Tsilioni I, Patel AB, Pantazopoulos H, Berretta S, Conti P, Leeman SE, et al. IL-37 Is Increased in Brains of Children With Autism Spectrum Disorder and Inhibits Human Microglia Stimulated by Neurotensin. Proc Natl Acad Sci USA (2019) 116(43):21659-65. doi: 10.1073/pnas.1906817116

93. Luo P, Feng C, Jiang C, Ren X, Gou L, Ji P, et al. IL-37b Alleviates Inflammation in the Temporomandibular Joint Cartilage Via IL-1R8 Pathway. Cell Prolif (2019) 52(6):e12692. doi: 10.1111/cpr.12692

94. Kacem O, Kaabachi W, Dhifallah IB, Hamzaoui A, Hamzaoui K. Elevated Expression of TSLP and IL-33 in Behcet's Disease Skin Lesions: IL-37 Alleviate Inflammatory Effect of TSLP. Clin Immunol (2018) 192:14-9. doi: 10.1016/j.clim.2018.03.016

95. Ozguclu S, Duman T, Ates FSO, Kucuksahin O, Colak S, Olmez U. Serum Interleukin-37 Level and Interleukin-37 Gene Polymorphism in Patients With Behcet Disease. Clin Rheumatol (2019) 38(2):495-502. doi: 10.1007/ s10067-018-4288-7

96. Jing L, Kim S, Sun L, Wang L, Mildner E, Divaris K, et al. IL-37- and IL-35/ IL-37-Producing Plasma Cells in Chronic Periodontitis. J Dent Res (2019) 98 (7):813-21. doi: 10.1177/0022034519847443

97. Kim MS, Baek AR, Lee JH, Jang AS, Kim DJ, Chin SS, et al. IL-37 Attenuates Lung Fibrosis by Inducing Autophagy and Regulating TGF-Betal Production in Mice. J Immunol (2019) 203(8):2265-75. doi: 10.4049/jimmunol.1801515

98. Wang L, Liu Z, Huang D, Ran Y, Zhang H, He J, et al. IL-37 Exerts AntiInflammatory Effects in Fetal Membranes of Spontaneous Preterm Birth Via the NF-kappaB and IL-6/STAT3 Signaling Pathway. Mediators Inflamm (2020) 2020:1069563. doi: 10.1155/2020/1069563

99. Li T, Li H, Li W, Chen S, Feng T, Jiao W, et al. Interleukin-37 Sensitize the Elderly Type 2 Diabetic Patients to Insulin Therapy Through Suppressing the Gut Microbiota Dysbiosis. Mol Immunol (2019) 112:322-9. doi: 10.1016/ j.molimm.2019.06.008

100. Wang P, Wang H, Li C, Zhang X, Xiu X, Teng P, et al. Dysregulation of microRNA-657 Influences Inflammatory Response Via Targeting
Interleukin-37 in Gestational Diabetes Mellitus. J Cell Physiol (2019) 234 (5):7141-8. doi: $10.1002 / j c p .27468$

101. Kapelouzou A, Kontogiannis C, Tsilimigras DI, Georgiopoulos G, Kaklamanis L, Tsourelis L, et al. Differential Expression Patterns of Toll Like Receptors and Interleukin-37 Between Calcific Aortic and Mitral Valve Cusps in Humans. Cytokine (2019) 116:150-60. doi: 10.1016/j.cyto.2019.01.009

102. Zeng Q, Song R, Fullerton DA, Ao L, Zhai Y, Li S, et al. Interleukin-37 Suppresses the Osteogenic Responses of Human Aortic Valve Interstitial Cells In Vitro and Alleviates Valve Lesions in Mice. Proc Natl Acad Sci USA (2017) 114(7):1631-6. doi: 10.1073/pnas.1619667114

103. Zhan Q, Zeng Q, Song R, Zhai Y, Xu D, Fullerton DA, et al. IL-37 Suppresses MyD88-Mediated Inflammatory Responses in Human Aortic Valve Interstitial Cells. Mol Med (2017) 23:83-91. doi: 10.2119/molmed.2017.00022

104. Huang N, Liu K, Liu J, Gao X, Zeng Z, Zhang Y, et al. Interleukin-37 Alleviates Airway Inflammation and Remodeling in Asthma Via Inhibiting the Activation of NF- $\mathrm{KB}$ and STAT3 Signalings. Int Immunopharmacol (2018) 55:198-204. doi: 10.1016/j.intimp.2017.12.010

105. Meng P, Chen ZG, Zhang TT, Liang ZZ, Zou XL, Yang HL, et al. IL-37 Alleviates House Dust Mite-Induced Chronic Allergic Asthma by Targeting TSLP Through the NF-KB and ERK1/2 Signaling Pathways. Immunol Cell Biol (2019) 97(4):403-15. doi: 10.1111/imcb.12223

106. Lv J, Xiong Y, Li W, Cui X, Cheng X, Leng Q, et al. IL-37 Inhibits IL-4/IL-13Induced CCL11 Production and Lung Eosinophilia in Murine Allergic Asthma. Allergy (2018) 73(8):1642-52. doi: 10.1111/all.13395

107. Zhu J, Dong J, Ji L, Jiang P, Leung TF, Liu D, et al. Anti-Allergic Inflammatory Activity of Interleukin-37 Is Mediated by Novel Signaling Cascades in Human Eosinophils. Front Immunol (2018) 9:1445. doi: 10.3389/fimmu.2018.01445

108. Li A, Ling Y, Song Z, Cheng X, Ding L, Jiang R, et al. Correlation Between Early Plasma Interleukin 37 Responses With Low Inflammatory Cytokine Levels and Benign Clinical Outcomes in Severe Acute Respiratory Syndrome Coronavirus 2 Infection. J Infect Dis (2021) 223(4):568-80. doi: 10.1093/ infdis/jiaa713

109. Conti P, Ronconi G, Caraffa A, Gallenga CE, Ross R, Frydas I, et al. Induction of Pro-Inflammatory Cytokines (IL-1 and IL-6) and Lung Inflammation by Coronavirus-19 (COVI-19 or SARS-CoV-2): Anti-Inflammatory Strategies. J Biol Regul Homeost Agents (2020) 34(2):327-31. doi: 10.23812/CONTI-E

110. Conti P, Caraffa A, Gallenga CE, Ross R, Kritas SK, Frydas I, et al. Coronavirus- 19 (SARS-CoV-2) Induces Acute Severe Lung Inflammation Via IL-1 Causing Cytokine Storm in COVID-19: A Promising Inhibitory Strategy. J Biol Regul Homeost Agents (2020) 34(6):1971-5. doi: 10.23812/20-1-E

111. Hojen JF, Rasmussen TA, Andersen KL, Winckelmann AA, Laursen RR, Gunst JD, et al. Interleukin-37 Expression Is Increased in Chronic HIV-1Infected Individuals and Is Associated With Inflammation and the Size of the Total Viral Reservoir. Mol Med (2015) 21:337-45. doi: 10.2119/ molmed.2015.00031

112. Samarani S, Abulkhir A, Amre D, Mehraj V, Tremblay C, Routy JP, et al. The Anti-Inflammatory IL-37/SIGIRR Axis Is Functionally Compromised in HIV Infection. AIDS (2019) 33(11):1693-703. doi: 10.1097/QAD. 0000000000002271

113. Mountford S, Effenberger M, Noll-Puchta H, Griessmair L, Ringleb A, Haas S, et al. Modulation of Liver Inflammation and Fibrosis by Interleukin-37. Front Immunol (2021) 12:603649. doi: 10.3389/fimmu.2021.603649

114. La Rosa F, Agostini S, Saresella M, Costa AS, Piancone F, Miglioli R, et al. Deregulation of IL-37 and Its miRNAs Modulators in Sarcopenic Patients After Rehabilitation. J Transl Med (2021) 19(1):172. doi: 10.1186/s12967021-02830-5

Conflict of Interest: The authors declare that the research was conducted in the absence of any commercial or financial relationships that could be construed as a potential conflict of interest.

Copyright (0) 2021 Su and Tao. This is an open-access article distributed under the terms of the Creative Commons Attribution License (CC BY). The use, distribution or reproduction in other forums is permitted, provided the original author(s) and the copyright owner(s) are credited and that the original publication in this journal is cited, in accordance with accepted academic practice. No use, distribution or reproduction is permitted which does not comply with these terms. 